\title{
Standard Tensorial Analysis of Local Ordering in Proteins from Residual Dipolar Couplings
}

\author{
Eva Meirovitch, ${ }^{* \dagger}{ }^{\dagger}$ Donghan Lee, ${ }^{\ddagger}$ Korvin F. A. Walter, ${ }^{\ddagger}$ and Christian Griesinger ${ }^{\ddagger}$ \\ ${ }^{\dagger}$ The Mina and Everard Goodman Faculty of Life Sciences, Bar-Ilan University, Ramat-Gan 52900, Israel \\ ${ }^{\ddagger}$ Department of NMR-based Structural Biology, Max-Planck Institute for Biophysical Chemistry, Göttingen, Germany
}

ABSTRACT: Residual dipolar couplings (RDCs) in proteins arise from independent external medium-related and internal protein-related ordering of the spin-bearing probe. Griesinger et al. developed a method for treating RDCs in proteins. The global ordering is given in the standard manner by a rank 2 tensor specified in a known molecular frame, MF. The local ordering is described by the spherical harmonic ensemble averages, $\left\langle Y_{2 m}(\theta, \varphi)\right\rangle, m=0, \pm 1, \pm 2$, also given in MF. From these quantities, a method we call mf-RDC derives the squared generalized order parameter $\left(S_{\mathrm{rdc}}^{2}\right)$, the amplitude (direction)

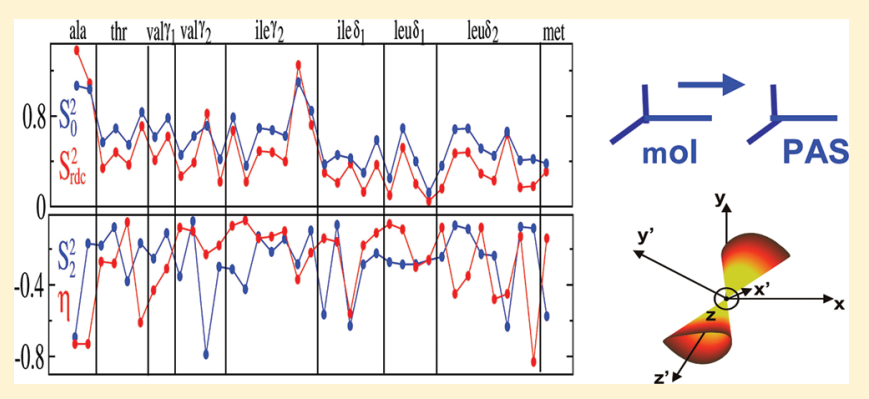
of the anisotropic disorder, $\eta\left(\bar{\Phi}^{\prime}\right)$, and an approximation, $(\mathrm{N}-\mathrm{H})_{\mathrm{eff}}$ to the average probe orientation, i.e., to the local director. $(\mathrm{N}-\mathrm{H})_{\mathrm{eff}}$ is determined through a frame transformation where $\left\langle Y_{20}\right\rangle$ is maximized. $\bar{\Phi}^{\prime}$ is associated with a subsequent frame transformation where $\left\langle Y_{22}+Y_{2-2}\right\rangle$ is maximized. The mfRDC method was applied previously to $\mathrm{N}-\mathrm{H}$ and $\mathrm{C}-\mathrm{C}_{\text {methyl }}$ sites in ubiquitin. In this study, we convert the respective $\left\langle Y_{2 m}(\theta, \varphi)\right\rangle$ 's into a Saupe tensor, which is diagonalized. This is the standard procedure. It yields the eigenvalues, $S_{x x}, S_{y y}$, and $S_{z z}$ and the Principal Axis System (PAS) of the rank 2 local ordering tensor, $S_{1} . S_{\text {rdc }}^{2}, \eta$, and $\bar{\Phi}^{\prime}$ can be recast as $S_{x x}, S_{y y}$, and $S_{z z}$. The mf-RDC frame transformations are not the same as the conventional Wigner rotation. The standard tensorial analysis provides new information. The contribution of local ordering rhombicity to $S_{\text {rdc }}{ }^{2}$ is evaluated. For the $\alpha$-helix of ubiquitin, the main local ordering axis is assigned as $\mathrm{C}_{i-1}^{\alpha}-\mathrm{C}_{i}^{\alpha}$; for the methyl sites, it is associated with the $\mathrm{C}-\mathrm{C}_{\text {methyl }}$ axis, as in mf-RDC. Ordering strength correlates with methyl type. The strength (rhombicity) of $S_{1}$ associated with picosecond-nanosecond local motions is reduced moderately (substantially) by nanosecond-millisecond local motions. A scheme for analyzing experimental RDCs based on the standard tensorial perspective, which allows for arbitrary orientation of the local director in the protein and of the PAS of $S_{1}$ in the probe, is formulated.

\section{INTRODUCTION}

Residual Dipolar Couplings (RDCs) between pairs of NMR nuclei in spatial proximity arise when the protein containing them is dissolved in an anisotropic liquid-crystalline (LC) medium. ${ }^{1,2}$ The RDC has emerged as an important factor in 3D NMR structure determination procedures, a useful means for detecting the mobility of large chain segments such as domains, and an important source for the detection of slow local motions. ${ }^{3}$ Effort has been invested in recent years to develop methods for accurate determination of the global ordering (alignment) tensors ${ }^{3}$ and for minimizing structural noise. ${ }^{4}$ Significant advances in perfecting experimental methodologies and establishing theoretical approaches for obtaining from RDCs accurate information on the dynamic structure of the protein have been reported. $^{3-24}$

In the weakly ordered media used to measure RDCs from proteins, motions faster than approximately $10 \mathrm{~ms}$ affect the RDC. ${ }^{3}$ NMR spin relaxation is probing picosecond-nanosecond motions extending up to the (nanosecond) rotational correlation time of the protein. ${ }^{25} \mathrm{NMR}$ relaxation dispersion is probing motions extending from approximately $50 \mu$ s to approximately $10 \mathrm{~ms}^{26}$ Thus, the RDC is unique in providing information on motions that are slower than the rotational correlation time of the protein and are not detected by relaxation dispersion methods. It should be pointed out that RDCs report exclusively on averaged out motional effects that materialize as spatial restrictions, i.e., as features of (global and local) ordering. The ${ }^{15} \mathrm{~N}-{ }^{1} \mathrm{H}$ bond is useful for studying local ordering at protein backbone sites, and the $\mathrm{C}-{ }^{13} \mathrm{CH}_{3}$ moiety (which for fast methyl rotation is represented by the $\mathrm{C}-\mathrm{C}_{\text {methyl }}$ axis) is useful for studying local ordering in protein side chains. Herein we focus on these probes.

RDCs arise due to nonuniform sampling of the conformational space. For rigid molecules, this is due to the anisotropic liquid-crystal (LC) solvent, which exerts spatial restrictions at the site of their motion. ${ }^{27,28}$ In the standard theories for treating restricted motions in liquids, ${ }^{27,28}$ these constraints are expressed in terms of second-rank ordering tensors, $\mathbf{S}_{\mathrm{g}}$. The eigenvalues of these tensors, i.e., the order parameters, are defined in terms of the potential of mean torque, $\mathrm{POMT}_{\mathrm{g}}$. The latter determines the probability distribution function, $P_{\text {eq,g, }}$, which describes the orientation of the molecule relative to

Received: February 13, 2012

Revised: April 13, 2012

Published: April 18, 2012 
the LC director. ${ }^{27,28}$ For small and rigid molecules, methods for determining $S_{\mathrm{g}}$ tensors and associated geometric features from experimental RDCs were developed in early work. ${ }^{27-32}$ These methods have been extended to rigid proteins. ${ }^{3}$

When the molecule is flexible, the motion of the probe is restricted not only by the external anisotropic medium but also by its immediate (intramolecular) protein surroundings. For independent external and internal POMTs, the analysis of RDCs from small flexible molecules is outlined formally in ref 29. Along this line of thought, and with a model-free perspective, Griesinger and co-workers developed a method called Model Free Analysis (MFA) ${ }^{4,10}$ for analyzing RDCs from proteins. The global ordering is treated in the standard manner in terms of a diagonal rank 2 tensor, $S_{g}$, defined with respect to the uniaxial liquid-crystal (LC) director. Its orientation is specified with respect to the $3 \mathrm{D}$ NMR or X-ray crystallography structure of the protein (MF). The local ordering is treated using a procedure we call $\mathrm{mf}-\mathrm{RDC}$. This scheme was applied extensively to $\mathrm{N}-\mathrm{H}$ sites in ubiquitin ${ }^{4-12,18}$ and recently also to $\mathrm{C}-\mathrm{C}_{\text {methyl }}$ sites in this protein. ${ }^{13}$ We refer to "sites" rather than "bonds" to account for the fact that the symmetry of the RDC probe (hence the symmetry of the local ordering) is allowed to be rhombic. For $\mathrm{N}-\mathrm{H}$ sites, the MF frame is associated with the $\mathrm{N}-\mathrm{H}$-bondcomprising peptide-bond plane. For methyl sites, it is associated with the plane defined by the $\mathrm{C}_{i-1}-\mathrm{C}_{i}$ bond and the $\mathrm{C}_{i}-\mathrm{C}_{\text {methyl }}$ axis. Reference 4 used $2003 \mathrm{~N}-\mathrm{H}$ RDCs obtained from 36 different alignment media. Reference 13 used 605 methyl group $\mathrm{RDCs}$ obtained from 13 different alignment media. These date are used in this study.

As pointed out above, it is assumed in mf-RDC that the external and internal ordering potentials are independent. Hence, the associated ordering phenomena may be treated independently. Furthermore, the local ordering may be considered the same in all of the LC media used. Taking the local director uniaxial, the spherical harmonic ensemble averages, $\left\langle Y_{2 m}\left(\theta^{\prime \prime}, \varphi^{\prime \prime}\right)\right\rangle$, $m=0, \pm 1, \pm 2$, are derived in the MF frame as representatives of the local ordering (a biaxial local director would require 25 ensemble averages featuring the Wigner rotation matrix elements $D_{k m}^{2}$, where $\left.k, m=0, \pm 1, \pm 2\right)$. This requires at least five LC media that yield different $\boldsymbol{S}_{\mathbf{g}}$ tensors. From the $\left\langle Y_{2 m}(\theta, \varphi)\right\rangle$ 's, one derives the squared generalized order parameter, $S_{\mathrm{rdc}}{ }^{2}$. The $(\theta, \varphi)$ frame is transformed into the $\left(\theta^{\prime}, \varphi^{\prime}\right)$ frame, where an effective $\mathrm{N}-\mathrm{H}$ orientation, $(\mathrm{N}-\mathrm{H})_{\text {eff, }}$ given in MF by the polar angles $\left(\theta_{\text {eff }}, \varphi_{\text {eff }}\right)$, is determined by maximizing $\left\langle Y_{20}\left(\theta^{\prime}, \varphi^{\prime}\right)\right\rangle$. This procedure assumes that the $\mathrm{N}-\mathrm{H}$ distribution is axially symmetric. $(\mathrm{N}-\mathrm{H})_{\text {eff }}$ is thus an approximation to the average $\mathrm{N}-\mathrm{H}$ orientation, $(\mathrm{N}-\mathrm{H})_{\text {av }}$, which represents the local director. The amplitude of the anisotropic disorder, $\eta$, is defined in the $\left(\theta^{\prime}, \varphi^{\prime}\right)$ frame. ${ }^{6}$ By a specific rotation $\bar{\Phi}^{\prime}$ around its $Y$-axis, ${ }^{10}$ the $\left(\theta^{\prime}, \varphi^{\prime}\right)$ frame is transformed into the $\left(\theta^{\prime \prime}, \varphi^{\prime \prime}\right)$ frame, where $\left\langle Y_{22}\left(\theta^{\prime \prime}, \varphi^{\prime \prime}\right)+Y_{2-2}\left(\theta^{\prime \prime}, \varphi^{\prime \prime}\right)\right\rangle$ is at maximum. The $\left(\theta^{\prime \prime}, \varphi^{\prime \prime}\right)$ frame represents the PAS of the $S_{1}$ tensor. The angle $\bar{\Phi}^{\prime}$ represents the direction of the anisotropic disorder.

In this study, we convert the $\left\langle Y_{2 m}\right\rangle$ 's obtained previously with MFA for each $(\mathrm{N}-\mathrm{H})$ (ref 4) and $\mathrm{C}-\mathrm{C}_{\text {methyl }}$ (ref 13) site in ubiquitin into the corresponding Saupe tensor, which is diagonalized. This yields the eigenvalues $S_{x x}, S_{y y}$, and $S_{z z}$ and the Principal Axis System (PAS) of the local ordering tensor, $S_{1} . S_{0}^{2}=S_{z z}$ represents the strength of the local ordering, and $S_{2}^{2}=(2 / 3)^{1 / 2}\left(S_{x x}-S_{y y}\right)$ represents its rhombicity $\left(S_{0}^{2}\right.$ and $S_{2}^{2}$ are the Wigner scheme order parameters); the PAS axes represent orientations of preferential ordering.
We find that $S_{\text {rdc }}^{2}, \eta$, and $\bar{\Phi}^{\prime}$ can be recast as $S_{x x}, S_{y y}$, and $S_{z z}$. The angles $\theta_{\text {eff }} \varphi_{\text {eff }}$ and $\bar{\Phi}^{\prime}$, on the one hand, and the Euler angles of the conventional Wigner rotation, on the other hand, are not the same. The following new information is obtained by pursuing the standard tensorial analysis. The contribution of local ordering rhombicity to $S_{\text {rdc }}{ }^{2}$ is evaluated quantitatively. For the $\alpha$-helix of ubiquitin, the main local ordering axis is assigned as $\mathrm{C}_{i-1}^{\alpha}-\mathrm{C}_{i}^{\alpha}$. For the $\mathrm{C}-\mathrm{C}_{\text {methyl }}$ sites of ubiquitin, the main local ordering axis is associated with the $\mathrm{C}-\mathrm{C}_{\text {methyl }}$ axis. The strength of the local ordering correlates with methyl type/side-chain length. Local ordering tensors from ${ }^{15} \mathrm{~N}-{ }^{1} \mathrm{H}$ spin relaxation and ${ }^{15} \mathrm{~N}-{ }^{1} \mathrm{H}$ RDC are compared. We find that the strength (rhombicity) of the local ordering emerging from fast picosecond-nanosecond local motions is reduced moderately (substantially) when slow nanosecond-millisecond local motions are also present.

Physical ordering tensors are defined with respect to the direction of preferred ordering, called director. Here, both $S_{1}$ and the mf$\mathrm{RDC}$ parameters are defined with respect to $(\mathrm{N}-\mathrm{H})_{\text {static }}$ which represents the $\mathrm{N}-\mathrm{H}$ bond orientation in the X-ray crystallography or NMR structures. ${ }^{5}$ (Note that the definition of $(\mathrm{N}-\mathrm{H})_{\text {eff }}$ is independent of the derivation of the $\mathrm{S}_{1}$ tensor and the mf-RDC parameters). For the $\mathrm{N}-\mathrm{H}$ sites and the $\mathrm{C}-\mathrm{C}_{\text {methyl }}$ sites associated with relatively short side chains, using $(\mathrm{N}-\mathrm{H})_{\text {static }}$ instead of the local director is a reasonably good approximation (see below). However, a general scheme that determines inherently the orientation of the local director in the protein, which is the main structural/ geometric information sought in RDC research, is undoubtedly desirable. Allowing for arbitrary orientation of the local ordering frame with respect to the dipolar frame is also desirable. Given that typically the orientation of the dipolar frame in the probe is known, this will make possible assigning the local ordering frame from a structural point of view. We propose below such a scheme.

\section{THEORETICAL BACKGROUND FOR RDC ANALYSIS}

2.1. Comprehensive Tensorial Approach. The frame scheme underlying the tensorial approach proposed is depicted in Figure 1a. LF is the space-fixed laboratory frame with its $Z$-axis parallel to the external magnetic field. The uniaxial liquid crystal director points along LF. AF is the Principal Axis System (PAS) of the global ordering tensor, $\mathbf{S}_{\mathrm{g}}$. MF is a known molecular frame, typically the $3 \mathrm{D}$ NMR or X-ray crystallography structure. The PAS of AF is determined relative to MF. VF is the uniaxial local director given in the present case by $(\mathrm{N}-\mathrm{H})_{\mathrm{av}}$ or $\left(\mathrm{C}-\mathrm{C}_{\text {methyl }}\right)_{\mathrm{av}}$. The AF, MF, and VF frames are fixed in the protein. OF is the PAS of the local ordering tensor, $S_{1}$. DF is the PAS of the ${ }^{15} \mathrm{~N}-{ }^{1} \mathrm{H}$ dipolar tensor. OF and DF are fixed in the probe. The PAS of OF is determined relative to the DF frame.

Figure $1 \mathrm{~b}$ depicts the various frames in the context of a typical protein structure. Part a shows the $Z$-axes of the global ordering frame (AF) and molecular frame (MF). Part b shows a segment of the central helix with a representative $\mathrm{N}-\mathrm{H}$ bond defined as $\mathrm{Z}_{\mathrm{MF}}$ (local). The local director, $\mathrm{VF}$, is depicted as $(\mathrm{N}-\mathrm{H})_{\mathrm{eq}}$, which according to the $\mathrm{mf}-\mathrm{RDC}$ nomenclature is $(\mathrm{N}-\mathrm{H})_{\mathrm{av}}$. Part $\mathrm{c}$ shows this $\mathrm{N}-\mathrm{H}$ bond, pointing along the (axial) dipolar frame, DF, in the context of the respective peptide-bond plane, represented by the $C_{i-1}^{\alpha}-C_{i}^{\alpha}$ axis. This axis is identified with the main local ordering axis, $\mathrm{Z}_{\mathrm{OF}}$ (see below).

The contribution of the dipolar interaction between two nuclei, $i$ and $j$, to the spin Hamiltonian is given by the following expression $^{27,28,32}$

$$
H_{i j, \mathrm{DF}}=\sum_{m, k}\left\langle D_{m k}^{2}\left(\Omega_{\mathrm{LF}-\mathrm{DF}}\right)\right\rangle F_{i j, \mathrm{DF}}^{(2, k) *} T_{i j, \mathrm{LF}}^{(2, m)}
$$


(a)
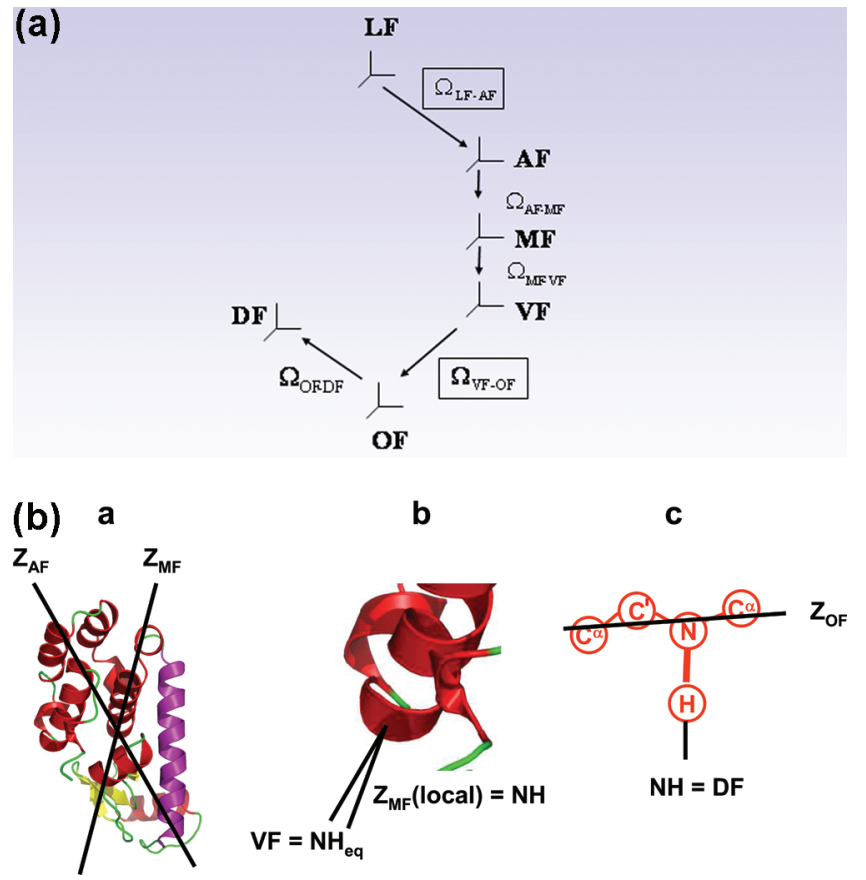

Figure 1. (a) Frame scheme of the standard tensorial analysis. LF, laboratory frame, with its $Z$-axis along the external magnetic field; $A F$, PAS of the global ordering tensor, $\mathbf{S}_{\mathrm{g}}$; MF, known molecular frame, typically the X-ray crystallography or average NMR structure; VF, local director frame; OF, PAS of the local ordering tensor, $\mathbf{S}_{\mathrm{l}}$, DF, PAS of the magnetic ${ }^{15} \mathrm{~N}-{ }^{1} \mathrm{H}$ dipolar tensor. $\mathrm{AF}, \mathrm{MF}$, and $\mathrm{VF}$ are fixed in the protein; OF and DF are fixed in the probe. The Euler angles $\Omega_{\mathrm{LF}-\mathrm{AF}}$ are distributed due to the external ordering. The Euler angles $\Omega_{\mathrm{VF}-\mathrm{OF}}$ are distributed due to the local ordering. (b) The frames of (a), shown in the context of a typical protein structure. $Z$-axes of the global ordering (AF) and molecular (MF) frames (part a). A segment of the central helix with a specific $\mathrm{N}-\mathrm{H}$ bond defined as $\mathrm{Z}_{\mathrm{MF}}$ (local). The local director, $\mathrm{VF}$, is depicted as $(\mathrm{N}-\mathrm{H})_{\mathrm{eq}}$, which according to the $\mathrm{mf}-\mathrm{RDC}$ nomenclature is $(\mathrm{N}-\mathrm{H})_{\mathrm{av}}$ (part $\left.\mathrm{b}\right)$. Representative $\mathrm{N}-\mathrm{H}$ bond, pointing along the (axial) dipolar frame, DF, shown in the context of the respective peptide-bond plane, represented by the $C_{i-1}^{\alpha}-C_{i}^{\alpha}$ axis. This axis is identified with the main local ordering axis, $\mathrm{Z}_{\mathrm{OF}}$ (part c).

where $F_{i j, D F}^{(2, k)}$ denotes the components of the magnetic dipolar tensor in the DF frame, and $T_{\mathrm{L}}^{(2, m)}$ denotes the components of the relevant spin operators in the LF frame. $D_{k m}^{2}$ are the Wigner rotation matrix elements.

For rigid proteins, $\Omega_{\mathrm{LF}-\mathrm{DF}}=\Omega_{\mathrm{LF}-\mathrm{AF}}+\Omega_{\mathrm{AF}-\mathrm{DF}}$. That is, $\Omega_{\mathrm{LF}-\mathrm{DF}}$ represents the combined effects of rotations by both sets of Euler angles on the right of this equation (with $\Omega_{\mathrm{AF}-\mathrm{DF}}$ fixed). Averaging the appropriate trigonometric functions over the Euler angles $\Omega_{\mathrm{LF}-\mathrm{AF}}$ yields the global order parameters. Given that $\mathrm{Z}_{\mathrm{DF}}$ points along the $\mathrm{N}-\mathrm{H}$ bond, the Euler angles $\Omega_{\mathrm{AF}-\mathrm{DF}}$ represent the structural/geometric information of interest in RDC research. For a uniaxial LC and at least $C_{2 v}$ symmetry around the $Z$-axis of AF, the only Wigner scheme order parameters that survive are $S_{\mathrm{g} 0}^{2} \equiv\left\langle D_{00}^{2}\left(\Omega_{\mathrm{LF}-\mathrm{AF}}\right)\right\rangle$ and $S_{\mathrm{g} 2}^{2}$ $\equiv\left\langle D_{02}^{2}\left(\Omega_{\mathrm{LF}-\mathrm{AF}}\right)+D_{0-2}^{2}\left(\Omega_{\mathrm{LF}-\mathrm{AF}}\right)\right\rangle^{27,28,32}$ The corresponding Saupe scheme order parameters are $S_{\mathrm{g}, x x}=\left((3 / 2)^{1 / 2} S_{\mathrm{g} 2}^{2}-S_{\mathrm{g} 0}^{2}\right) / 2$, $S_{\mathrm{g}, y y}=-\left((3 / 2)^{1 / 2} S_{\mathrm{g} 2}^{2}+S_{\mathrm{g} 0}^{2}\right) / 2$, and $S_{\mathrm{g}, z z}=S_{\mathrm{g} 0 .}^{2}{ }^{27,28,32}$

The RDC between the nuclei $i$ and $j$ is given by ${ }^{3}$

$$
\begin{aligned}
\mathrm{RDC}_{i j}= & \left(\mu_{0} / 4 \pi\right) \gamma_{i} \gamma_{j} h /\left(4 \pi^{2} r_{i j}^{3}\right)\left[S_{\mathrm{g} 0}^{2} P_{2}\left(\cos \beta_{\mathrm{AF}-\mathrm{DF}}\right)\right. \\
& +\sqrt{3 / 2}\left[S_{\mathrm{g} 2}^{2} \sin ^{2}\left(\beta_{\mathrm{AF}-\mathrm{DF}}\right) \cos \left(2 \alpha_{\mathrm{AF}-\mathrm{DF}}\right)\right]
\end{aligned}
$$

where $\mu_{0}$ is the permeability of vacuum; $\gamma_{i}$ and $\gamma_{j}$ are the magnetogyric ratios of the nuclei $i$ and $j$; $h$ is Planck's constant; and $r_{i j}$ is the distance between the nuclei $i$ and $j$.

In the presence of local ordering, one has $\Omega_{\mathrm{LF}-\mathrm{DF}}=\Omega_{\mathrm{LF}-\mathrm{AF}}+$ $\Omega_{\mathrm{AF}-\mathrm{MF}}+\Omega_{\mathrm{MF}-\mathrm{VF}}+\Omega_{\mathrm{VF}-\mathrm{OF}}+\Omega_{\mathrm{OF}-\mathrm{DF}}$ (Figure 1 ). That is, $\Omega_{\mathrm{LF}-\mathrm{DF}}$ represents the combined effects of the rotations by all of the Euler angles on the right of this equation (with $\Omega_{\mathrm{AF}-\mathrm{MF}}$, $\Omega_{\mathrm{MF}-\mathrm{VF}}$, and $\Omega_{\mathrm{OF}-\mathrm{DF}}$ fixed). By virtue of the assumption that the $\mathrm{POMT}_{\mathrm{g}}$ and the $\mathrm{POMT}_{1}$ are independent, ${ }^{29}$ one may average separately over $\Omega_{\mathrm{LF}-\mathrm{AF}}$ to obtain $S_{\mathrm{g} 0}^{2}$ and $S_{\mathrm{g} 2}^{2}$ and over $\Omega_{\mathrm{VF}-\mathrm{OF}}$ to obtain $S_{\mathrm{lo}}^{2}$ and $S_{\mathrm{l} 2}^{2}$ (the index "l" is omitted below).

Let us delineate the basics of the standard tensorial approach as applied to the local ordering. This treatment is formally the same as the treatment of the global ordering. The $\mathrm{POMT}_{1}$ is denoted $u\left(\Omega_{\mathrm{VF}-\mathrm{OF}}\right)$ (in units of $k_{\mathrm{B}} T$ ). This function is expanded in the basis set of the Wigner rotation matrix elements. When only the lowest order, $L=2$, terms are preserved, one obtains ${ }^{27,28,32}$

$$
\begin{aligned}
u\left(\Omega_{\mathrm{VF}-\mathrm{OF}}\right)= & \frac{U\left(\Omega_{\mathrm{VF}-\mathrm{OF}}\right)}{k_{\mathrm{B}} T} \approx-c_{0}^{2} D_{0,0}^{2}\left(\Omega_{\mathrm{VF}-\mathrm{OF}}\right) \\
& -c_{2}^{2}\left[D_{0,2}^{2}\left(\Omega_{\mathrm{VF}-\mathrm{OF}}\right)+D_{0,-2}^{2}\left(\Omega_{\mathrm{VF}-\mathrm{OF}}\right)\right]
\end{aligned}
$$

The coefficient $c_{0}^{2}$ evaluates the strength of the $\mathrm{POMT}_{1}$, and the coefficient $c_{2}^{2}$ evaluates its rhombicity.

The equilibrium probability distribution associated with the local ordering is given by $P_{\mathrm{eq}, 1}=\exp \left[-U\left(\Omega_{\mathrm{VF}-\mathrm{OF}}\right) / k_{\mathrm{B}} T\right] /$ $<\exp \left[-U\left(\Omega_{\mathrm{VF}-\mathrm{OF}}\right) / k_{\mathrm{B}} T\right]>$, where $<\ldots>$ means averaging over $\Omega_{\mathrm{VF}-\mathrm{OF}}$.

The local order parameters are defined as ${ }^{27,28,32}$

$$
\begin{aligned}
\left\langle D_{0 m}^{2}\left(\Omega_{\mathrm{VF}-\mathrm{OF}}\right)\right\rangle= & \int \mathrm{d} \Omega_{\mathrm{VF}-\mathrm{OF}} D_{0 m}^{2}\left(\Omega_{\mathrm{VF}-\mathrm{OF}}\right) \\
& \times \exp \left[-u\left(\Omega_{\mathrm{VF}-\mathrm{OF}}\right)\right] / \int \mathrm{d} \Omega_{\mathrm{VF}-\mathrm{OF}} \exp \left[-u\left(\Omega_{\mathrm{VF}-\mathrm{OF}}\right)\right]
\end{aligned}
$$

For a uniaxial local director and at least $C_{2 v}$ symmetry around the $Z$-axis of the local ordering frame, OF, only $S_{0}^{2} \equiv$ $\left\langle D_{02}^{2}\left(\Omega_{\mathrm{VF}-\mathrm{OF}}\right)\right\rangle$ and $S_{2}^{2} \equiv\left\langle D_{02}^{2}\left(\Omega_{\mathrm{VF}-\mathrm{OF}}\right)+D_{0-2}^{2}\left(\Omega_{\mathrm{VF}-\mathrm{OF}}\right)\right\rangle$ survive. $^{27,28,32}$ The Saupe scheme order parameters relate to $S_{0}^{2}$ and $S_{2}^{2}$ as $S_{x x}=\left((3 / 2)^{1 / 2} S_{2}^{2}-S_{0}^{2}\right) / 2, S_{y y}=-\left((3 / 2)^{1 / 2} S_{2}^{2}+S_{0}^{2}\right) / 2$, and $S_{z z}=S_{0}^{2}$.

The RDC between the nuclei $i$ and $j$ that emerges from the combined effect of the independent global and local ordering phenomena is given by

$$
\begin{aligned}
\mathrm{RDC}_{i j}= & \left(\mu_{0} / 4 \pi\right) \gamma_{i} \gamma_{j} h /\left(4 \pi^{2} r_{i j}^{3}\right) \sum_{p, q, r}\left\langle D_{0 p}^{2}\left(\Omega_{\mathrm{LF}-\mathrm{AF}}\right)\right\rangle \\
& \times D_{p q}^{2}\left(\Omega_{\mathrm{AF}-\mathrm{MF}}\right) D_{q 0}^{2}\left(\Omega_{\mathrm{MF}-\mathrm{VF}}\right)\left\langle D_{0 r}^{2}\left(\Omega_{\mathrm{VF}-\mathrm{OF}}\right)\right\rangle \\
& \times D_{r 0}^{2}\left(\Omega_{\mathrm{OF}-\mathrm{DF}}\right)
\end{aligned}
$$

with the various constants defined above. Equation 5 refers to a particular RDC in a given LC medium.

This expression comprises five terms. The first term represents the eigenvalues of $\boldsymbol{S}_{\mathrm{g}}$. The second term represents the orientation of $S_{g}$ in MF. The third term represents the orientation of the local director in the protein. The fourth term represents the eigenvalues of the $S_{1}$ tensor. The fifth term represents the orientation of the local ordering frame with respect to the dipolar frame. All of these well-defined physical quantities can be determined with data fitting. Such a scheme comprises six variables for the local ordering (see below). By setting $\alpha_{\mathrm{OF}-\mathrm{DF}}$ equal to zero (which in our experience from ${ }^{15} \mathrm{~N}$ spin relaxation analysis with the slowly relaxing local structure 
(SRLS) approach is a reasonable approximation), ${ }^{33,34}$ the number of variables is reduced to 5 , as in mf-RDC. The important angles $\Omega_{\mathrm{MF}-\mathrm{VF}}=\left(\alpha_{\mathrm{MF}-\mathrm{VF}}, \beta_{\mathrm{MF}-\mathrm{VF}}, 0\right)$ and $\Omega_{\mathrm{OF}-\mathrm{DF}}=$ $\left(\alpha_{\mathrm{OF}-\mathrm{DF}}, \beta_{\mathrm{OF}-\mathrm{DF}}, 0\right)\left(\right.$ or $\left.\Omega_{\mathrm{OF}-\mathrm{DF}}=\left(0, \beta_{\mathrm{OF}-\mathrm{DF}}, 0\right)\right)$, which are not determined in the current scheme, are provided in the proposed scheme. The current scaling of the $\left\langle Y_{2 m}\right\rangle$ 's (ref 5), implied mainly by both $S_{g}$ and $S_{1}$ being defined with respect to the same frame $(M F)$, is unnecessary because now $S_{1}$ is defined with respect to VF, whereas $S_{g}$ is defined with respect to MF. The scheme proposed makes possible comparing local ordering tensors from RDC analysis and from spin relaxation analysis, provided that the method underlying the latter accounts for general tensorial properties. Currently the only method that fulfills this requirement is SRLS. ${ }^{33,34}$ Such comparison is more insightful than the current comparison of squared generalized order parameters, in particular in the context of elucidating the effect on nanosecond-millisecond local motions.

2.2. Actual Approach. 2.2.1. Global Ordering. The first two terms of eq $5, \sum_{p, q}\left\langle D_{0, p}^{2}\left(\Omega_{\mathrm{LF}-\mathrm{AF}}\right)\right\rangle D_{q 0}\left(\Omega_{\mathrm{AF}-\mathrm{MF}}\right)$, are treated separately in MFA.,10 The eigenvalues of $S_{g}$ $\left\langle D_{00}^{2}\left(\Omega_{\mathrm{LF}-\mathrm{AF}}\right)\right\rangle$ and $\left\langle D_{02}^{2}\left(\Omega_{\mathrm{LF}-\mathrm{AF}}\right)+D_{0-2}^{2}\left(\Omega_{\mathrm{LF}-\mathrm{AF}}\right)\right\rangle$, and the Euler angles, $\Omega_{\mathrm{AF}-\mathrm{MF}}$, which define the PAS of $\boldsymbol{S}_{\mathrm{g}}$ in the MF frame, are obtained as outlined in refs 4 and 10. It is assumed that the local ordering is independent of the LC medium. Using at least five different LC media (i.e., five different $\mathbf{S}_{\mathrm{g}}$ tensors), one derives the ensemble averages $\left\langle Y_{2 m}(\theta, \varphi)\right\rangle, m=$ $0, \pm 1, \pm 2$, that describe the local ordering. The analysis of these quantities within the scope of $\mathrm{mf}-\mathrm{RDC}$ is outlined in the next section.

2.2.2. Local Ordering: The MF-RDC Approach. As pointed out above, the MF frame is taken as the X-ray crystallography or NMR structure of the protein. We will use the designation "MF" to describe its local parts corresponding to the site of the $\mathrm{RDC}$ probe, which are relevant for treating the local ordering. For the $\mathrm{N}-\mathrm{H}$ sites, $\mathrm{Z}_{\mathrm{MF}}$ is parallel to the $\mathrm{N}-\mathrm{H}$ bond; $\mathrm{X}_{\mathrm{MF}}$ is perpendicular to $Z_{M F}$ in the peptide-bond plane; and $Y_{M F}$ is perpendicular to both $\mathrm{Z}_{\mathrm{MF}}$ and $\mathrm{X}_{\mathrm{MF}}$, i.e., to the peptide-bond plane. For the methyl sites, $Z_{M F}$ is parallel to the $C-C_{\text {methyl }}$ axis; $\mathrm{X}_{\mathrm{MF}}$ is perpendicular to $\mathrm{Z}_{\mathrm{MF}}$ in the plane defined by the $\mathrm{C}_{i-1}-$ $\mathrm{C}_{i}$ bond and the $\mathrm{C}_{i}-\mathrm{C}_{\text {methyl }}$ axis; and $\mathrm{Y}_{\mathrm{MF}}$ is perpendicular to both $\mathrm{Z}_{\mathrm{MF}}$ and $\mathrm{X}_{\mathrm{MF}}$. Both mf-RDC and the standard analysis proposed use these $\left\langle Y_{2 m}(\theta, \varphi)\right\rangle$ 's, and these definitions for the (local) MF frame.

The frame scheme used to analyze the $\left\langle Y_{2 m}(\theta, \varphi)\right\rangle$ 's for the $\mathrm{N}-\mathrm{H}$ sites is shown in Figure 2a. An analogous scheme (not shown) is used to treat the $\mathrm{C}-\mathrm{C}_{\text {methyl }}$ sites, with $\mathrm{DF}$ (the dipolar tensor frame) replaced by $\mathrm{QF}$ (the quadrupolar tensor frame). The angles $(\theta, \varphi)$ are the coordinates of the $\mathrm{N}-\mathrm{H}$ bond in the (rhombic) MF frame. The squared generalized order parameter, $S_{\mathrm{rdc}}{ }^{2}$, is defined in this frame (although it may be defined in any frame).

The frame $(\theta, \varphi)$ represents the nondiagonal instantaneous local ordering frame of a given $\mathrm{N}-\mathrm{H}$ bond, which is a member in the local $\mathrm{N}-\mathrm{H}$ distribution. This frame is transformed into another instantaneous local ordering frame, $\left(\theta^{\prime}, \varphi^{\prime}\right)$, as follows. One assumes that the $\mathrm{N}-\mathrm{H}$ distribution is axially symmetric. In this case, there will be a particular $\left(\theta^{\prime}, \varphi^{\prime}\right)$ frame with its $Z$-axis at the maximum of the axial $\mathrm{N}-\mathrm{H}$ distribution. This $Z$-axis represents the effective local director, $(\mathrm{N}-\mathrm{H})_{\mathrm{eff}}$. The respective $\left(\theta^{\prime}, \varphi^{\prime}\right)$ values, denoted $\left(\theta_{\text {effi }}, \varphi_{\text {eff }}\right)$, define the orientation of $(\mathrm{N}-\mathrm{H})_{\text {eff }}$ in the MF frame. In general, the instantaneous local ordering frames, $\left(\theta^{\prime}, \varphi^{\prime}\right)$, have their $Z$-axes orienting

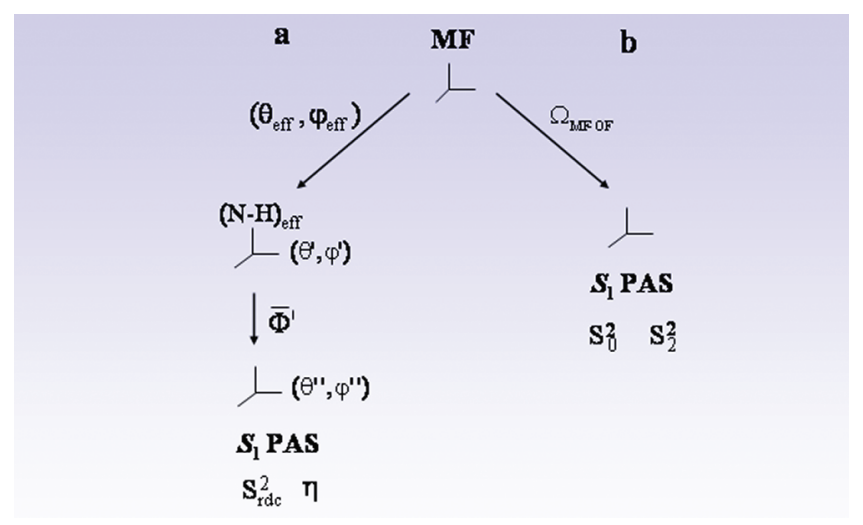

Figure 2. (a) Frame scheme of the mf-RDC method for treating the local ordering at $\mathrm{N}-\mathrm{H}$ sites. The frame scheme for treating the global ordering is depicted in Figure 1. The MF frame has $\mathrm{Z}_{\mathrm{MF}}$ parallel to the $\mathrm{N}-\mathrm{H}$ bond, $\mathrm{X}_{\mathrm{MF}}$ perpendicular to $\mathrm{Z}_{\mathrm{MF}}$ in the peptide-bond plane, and $\mathrm{Y}_{\mathrm{MF}}$ perpendicular to both $\mathrm{Z}_{\mathrm{MF}}$ and $\mathrm{X}_{\mathrm{MF}}$, i.e., to the peptide-bond plane. $\left(\theta_{\text {eff }}, \varphi_{\text {eff }}\right)$ are the coordinates of $(\mathrm{N}-\mathrm{H})_{\text {eff }}$ in the MF frame determined by maximizing $\left\langle Y_{20}\left(\theta^{\prime}, \varphi^{\prime}\right)\right\rangle$. The angles $\left(\theta^{\prime}, \varphi^{\prime}\right)$ are the coordinates of the $\mathrm{N}-\mathrm{H}$ bond in a local ordering frame with $\mathrm{Z}_{\mathrm{OF}}$ orienting preferentially along $(\mathrm{N}-\mathrm{H})_{\text {eff. }}$ The angles $\left(\theta^{\prime \prime}, \varphi^{\prime \prime}\right)$ are the coordinates of the $\mathrm{N}-\mathrm{H}$ bond in a local ordering frame where $\left\langle Y_{22}\left(\theta^{\prime \prime}, \varphi^{\prime \prime}\right)+Y_{2-2}\left(\theta^{\prime \prime}, \varphi^{\prime \prime}\right)\right\rangle$ is at maximum. The $\left(\theta^{\prime \prime}, \varphi^{\prime \prime}\right)$ frame is obtained by a rotation, $\bar{\Phi}^{\prime}$, given by eq 11 , around the $Y$-axis of the $\left(\theta^{\prime}, \varphi^{\prime}\right)$ frame. The $\left(\theta^{\prime \prime}, \varphi^{\prime \prime}\right)$ frame represents the PAS of the $\boldsymbol{S}_{1}$ tensor. The parameters $S_{\text {rdc }}{ }^{2}$ (eq 9), $\eta$ (eq 10), and $\bar{\Phi}^{\prime}$ (eq 11) characterize the local ordering. (b) Frame scheme of the standard tensorial method. The MF frame is defined above. $\Omega_{\mathrm{MF}-\mathrm{OF}}$ are the Euler angles associated with the conventional Wigner rotation from the MF frame to the PAS of $S_{1} \cdot S_{0}{ }^{2}$ and $S_{2}{ }^{2}$ are the Wigner scheme eigenvalues of $S_{1}$ (which yield in a straightforward manner the Saupe scheme eigenvalues; see text).

preferentially along $(\mathrm{N}-\mathrm{H})_{\text {eff }}$ Each $Z$-axis points along the corresponding instantaneous $\mathrm{N}-\mathrm{H}$ orientation, i.e., the corresponding principal axis of the dipolar tensor. This scenario is called Z-ordering (see below). The amplitude of the anisotropic disorder, $\eta$ (cf. eq 10 below), is defined in the $\left(\theta^{\prime}, \varphi^{\prime}\right)$ frame

The instantaneous frame, $\left(\theta^{\prime}, \varphi^{\prime}\right)$ is transformed into the instantaneous frame $\left(\theta^{\prime \prime}, \varphi^{\prime \prime}\right)$ where $\left\langle Y_{22}\left(\theta^{\prime \prime}, \varphi^{\prime \prime}\right)+Y_{2-2}\left(\theta^{\prime \prime}\right.\right.$, $\left.\left.\varphi^{\prime \prime}\right)\right\rangle$ is at maximum. This is accomplished by a rotation around the $Y$-axis of the $\left(\theta^{\prime}, \varphi^{\prime}\right)$ frame by an angle $\bar{\Phi}^{\prime}$ defined in eq 11 below. $\bar{\Phi}^{\prime}$ represents the direction of the anisotropic disorder.

The $\left(\theta^{\prime \prime}, \varphi^{\prime \prime}\right)$ frame represents the PAS of the local ordering tensor, $\mathbf{S}_{\mathrm{l}}$. We show this by recalling the following definitions 35

$$
\begin{aligned}
& \left\langle D_{02}^{2}(0, \theta, \varphi)+D_{0-2}^{2}(0, \theta, \varphi)\right\rangle \\
& \quad=\sqrt{4 \pi / 5}\left\langle Y_{02}(\theta, \varphi)+Y_{0-2}(\theta, \varphi)\right\rangle \\
& \left\langle D_{01}^{2}(0, \theta, \varphi)+D_{0-1}^{2}(0, \theta, \varphi)\right\rangle \\
& \quad=-\sqrt{4 \pi / 5}\left\langle Y_{01}(\theta, \varphi)+Y_{0-1}(\theta, \varphi)\right\rangle \\
& \left\langle D_{00}^{2}(0, \theta, \varphi)\right\rangle=\sqrt{4 \pi / 5}\left\langle Y_{20}(\theta, \varphi)\right\rangle \\
& \left\langle D_{01}^{2}(0, \theta, \varphi)-D_{0-1}^{2}(0, \theta, \varphi)\right\rangle \\
& \quad=-\sqrt{4 \pi / 5}\left\langle Y_{01}(\theta, \varphi)-Y_{0-1}(\theta, \varphi)\right\rangle \\
& \left\langle D_{02}^{2}(0, \theta, \varphi)-D_{0-2}^{2}(0, \theta, \varphi)\right\rangle \\
& \quad=\sqrt{4 \pi / 5}\left\langle Y_{02}(\theta, \varphi)-Y_{0-2}(\theta, \varphi)\right\rangle
\end{aligned}
$$




$$
\begin{aligned}
& \left\langle D_{00}^{2}\right\rangle=S_{z z} \\
& \left\langle D_{01}^{2}\right\rangle=\sqrt{2 / 3}\left(S_{x z}-i S_{y z}\right) \\
& \left\langle D_{02}^{2}\right\rangle=\sqrt{1 / 6} S_{x x}-\sqrt{1 / 6} S_{y y}-i \sqrt{2 / 3} S_{x y} \\
& \left\langle D_{0 m}^{2}\right\rangle=(-1)^{m}\left\langle D_{0-m}^{2}\right\rangle^{*} \\
& S_{z z}=\left\langle D_{00}^{2}\right\rangle \\
& S_{x x}=\sqrt{3 / 8}\left\langle D_{02}^{2}+D_{0-2}^{2}\right\rangle-\left\langle D_{00}^{2}\right\rangle / 2 \\
& S_{y y}=-\sqrt{3 / 8}\left\langle D_{02}^{2}+D_{0-2}^{2}\right\rangle-\left\langle D_{00}^{2}\right\rangle / 2 \\
& S_{x y}=S_{y x}=i \sqrt{3 / 8}\left\langle D_{02}^{2}-D_{0-2}^{2}\right\rangle \\
& S_{x z}=S_{z x}=\sqrt{3 / 8}\left\langle D_{01}^{2}-D_{0-1}^{2}\right\rangle \\
& S_{y z}=S_{z y}=i \sqrt{3 / 8}\left\langle D_{01}^{2}+D_{0-1}^{2}\right\rangle
\end{aligned}
$$

Maximizing $\left\langle Y_{22}\left(\theta^{\prime \prime}, \varphi^{\prime \prime}\right)+Y_{2-2}\left(\theta^{\prime \prime}, \varphi^{\prime \prime}\right)\right\rangle$ sets $\left\langle D_{01}^{2}+D_{0-1}^{2}\right\rangle$ and $\left\langle D_{01}^{2}-D_{0-1}^{2}\right\rangle$ equal to zero; ${ }^{6}$ hence, $S_{y z}$ and $S_{x z}$ in eq 8 are equal to zero. According to the definition of $\bar{\Phi}^{\prime}$ (eq 11), rotation around it sets $\bar{\Phi}^{\prime}$ equal to zero in the $\left(\theta^{\prime \prime}, \varphi^{\prime \prime}\right)$ frame; this sets $S_{x y}$ in eq 8 equal to zero. Thus, only the diagonal elements of $S_{1}$ survive.

The squared generalized order parameter, $S_{\mathrm{rdc}}{ }^{2}$, is defined by analogy with the squared generalized order parameter, $S^{2}$, used in model-free-based spin relaxation analysis. ${ }^{36}{S_{\mathrm{rdc}}}^{2}$ is given (in any frame) by ${ }^{5}$

$$
\begin{aligned}
S_{\mathrm{rdc}}^{2} & =\sum_{m=0, \pm 1, \pm 2}\left|\left\langle Y_{2 m}(\theta, \phi)\right\rangle\left\langle Y_{2 m}^{*}(\theta, \phi)\right\rangle\right| \\
m & = \pm 2, \pm 1,0
\end{aligned}
$$

Because the global ordering is determined without accounting for the local ordering, while both are defined with respect to the MF frame, it is necessary to scale the $\left\langle Y_{2 m}\right\rangle$ 's prior to calculating $S_{\text {rdc }}^{2}$, to compensate for having ignored the effect of the local ordering in determining the global ordering. Strategies for doing this have been developed. ${ }^{5,6}$ Similar to $S^{2}, 66$ the parameter $S_{\text {rdc }}^{2}$ is conceptualized as the amplitude of the local motion. 5 as ${ }^{5}$

The amplitude of the local motional anisotropy, $\eta$, is defined

$$
\eta=\left(\frac{\sum_{m= \pm 1, \pm 2}\left\langle Y_{2 \mathrm{~m}}\left(\theta^{\prime}, \varphi^{\prime}\right)\right\rangle\left\langle Y_{2 m}^{*}\left(\theta^{\prime}, \varphi^{\prime}\right)\right\rangle}{\sum_{m=0, \pm 1, \pm 2}\left\langle Y_{2 m}\left(\theta^{\prime}, \varphi^{\prime}\right)\right\rangle\left\langle Y_{2 m}^{*}\left(\theta^{\prime}, \varphi^{\prime}\right)\right\rangle}\right)^{1 / 2}
$$
as $^{6}$

The direction of the anisotropic local motion, $\bar{\Phi}^{\prime}$, is defined

$$
\bar{\Phi}^{\prime}=\frac{1}{2} \arctan \frac{\left\langle Y_{22}\left(\theta^{\prime}, \varphi^{\prime}\right)-Y_{2-2}\left(\theta^{\prime}, \varphi^{\prime}\right)\right\rangle}{i\left[\left\langle Y_{22}\left(\theta^{\prime}, \varphi^{\prime}\right)+Y_{2-2}\left(\theta^{\prime}, \varphi^{\prime}\right)\right\rangle\right]}
$$

The parameter $\left\langle Y_{20}\left(\theta^{\prime}, \varphi^{\prime}\right)\right\rangle$ represents the axial order. ${ }^{10}$ The parameters $\eta$ and $\bar{\Phi}^{\prime}$ represent the amount of anisotropic disorder. ${ }^{10}$ The direction of the $\mathrm{N}-\mathrm{C}^{\alpha}$ bond in the $\left(\theta^{\prime \prime}, \varphi^{\prime \prime}\right)$ frame is given by the angle $\xi_{\text {rdo }}^{\prime}$ defined as ${ }^{4}$

$$
\xi_{\mathrm{rdc}}^{\prime}=\arctan \frac{y_{\mathrm{NC} \alpha}^{\prime \prime}}{x_{\mathrm{NC} \alpha}^{\prime \prime}}
$$

This parameter characterizes the anisotropy with respect to the peptide-bond plane.
Formal analogies between the Wigner or Saupe scheme order parameters and the $\mathrm{mf}-\mathrm{RDC}$ parameters can be established. One has

$$
\begin{aligned}
& S_{10}^{2}=\sqrt{\left.{S_{\mathrm{rdc}}^{2}\left(1-\eta^{2}\right.}^{2}\right)} \\
& S_{\mathrm{l} 2}^{2}=\sqrt{2} \cos \left(2 \bar{\Phi}^{\prime}\right) \eta S_{\mathrm{rdc}}
\end{aligned}
$$

and

$$
\begin{aligned}
& S_{x x}=0.5\left[\sqrt{3 / 2}\left(\sqrt{2} \cos \left(2 \bar{\Phi}^{\prime}\right) \eta S_{\mathrm{rdc}}\right)-\sqrt{S_{\mathrm{rdc}}^{2}\left(1-\eta^{2}\right)}\right] \\
& S_{y y}=-0.5\left[\sqrt{3 / 2}\left(\sqrt{2} \cos \left(2 \bar{\Phi}^{\prime}\right) \eta S_{\mathrm{rdc}}\right)+\sqrt{{S_{\mathrm{rdc}}^{2}\left(1-\eta^{2}\right)}^{2}}\right. \\
& S_{z z}=\sqrt{{S_{\mathrm{rdc}}}^{2}\left(1-\eta^{2}\right)}
\end{aligned}
$$

2.2.3. Local Ordering: The Standard Approach. The local ordering is given by the last three terms of eq $5, \sum_{q, r}$ $D_{q 0}^{2}\left(\Omega_{\mathrm{MF}-\mathrm{VF}}\right)\left\langle D_{0 r}^{2}\left(\Omega_{\mathrm{VF}-\mathrm{OF}}\right)\right\rangle D_{r 0}^{2}\left(\Omega_{\mathrm{OF}-\mathrm{DF}}\right)$. The first term comprises the orientation of the local director in MF. The second term comprises the order parameters $S_{0}^{2}$ and $S_{2}^{2}$ associated with the diagonal $S_{1}$ tensor. The third term represents the relative orientation of the local ordering frame, OF, and the dipolar frame, DF. This scheme comprises the variables $\alpha_{\mathrm{MF}-\mathrm{VF}}$, $\beta_{\mathrm{MF}-\mathrm{VF}}, S_{0}^{2}, S_{2}^{2}, \alpha_{\mathrm{OF}-\mathrm{DF}}$, and $\beta_{\mathrm{OF}-\mathrm{DF}}$. On the basis of our experience with SRLS analysis of ${ }^{15} \mathrm{~N}$ spin relaxation in proteins, $\alpha_{\mathrm{OF}-\mathrm{DF}}$ may be set equal to zero. ${ }^{33,34}$ This reduces the number of variables for the local ordering to five, as in mf-RDC.

However, we do not use the expression depicted above for treating the local ordering. Rather, we use the expression $\left\langle D_{2 m}^{2}\left(\Omega_{\mathrm{MF}-\mathrm{OF}}\right)\right\rangle=(-1)^{m}(4 \pi / 5)^{1 / 2}\left\langle Y_{2 m}\right\rangle$. This is appropriate when (a) $\mathrm{VF}=\mathrm{Z}_{\mathrm{MF}}$ and (b) $\mathrm{Z}_{\mathrm{DF}}$ points along one of the principal axes of the local ordering frame, OF. With regard to point (a), there is evidence that $\mathrm{VF}$ and $\mathrm{Z}_{\mathrm{MF}}$ are near one another for the $\mathrm{N}-\mathrm{H}$ sites (Figure 4 of ref 10) and for the $\mathrm{C}-\mathrm{C}_{\text {methyl }}$ sites associated with short side chains (Figure $3 \mathrm{~b}$ of ref 13). With regard to point (b), the following is of interest. Maximizing $\left\langle Y_{20}\left(\theta^{\prime}, \varphi^{\prime}\right)\right\rangle$ is based on the approximation that $P_{\text {eq, }}$ is axially symmetric. In this context $\varphi^{\prime}=0$, and $P_{\text {eq, } 1}\left(\theta^{\prime}\right)$ may be expanded in the full basis set of the Legendre polynomials. ${ }^{37}$ When only the lowest order, $L=2$, term is preserved, one obtains $P_{\text {eq, } 1}\left(\theta^{\prime}\right) \sim\left\langle P_{2}\left(\cos \theta^{\prime}\right)\right\rangle=\left\langle Y_{20}\left(\theta^{\prime}, 0\right)\right\rangle$. Mazimizing this ensemble average is formally equivalent to minimizing a $\mathrm{POMT}_{1}$ given by the first term of eq 3 . In the $\left(\theta^{\prime \prime}, \varphi^{\prime \prime}\right)$ frame, $P_{\text {eq, }}$ is expanded in the full basis set of the spherical harmonic functions. When only the $L=2, K=0,2$, terms are preserved, one obtains $P_{\text {eq, } 1}\left(\theta^{\prime \prime}, \varphi^{\prime \prime}\right) \sim\left\langle Y_{20}\left(\theta^{\prime}, \varphi^{\prime}\right)\right\rangle+\left\langle Y_{22}\left(\theta^{\prime \prime}, \varphi^{\prime \prime}\right)+\right.$ $\left.Y_{2-2}\left(\theta^{\prime \prime}, \varphi^{\prime \prime}\right)\right\rangle$. Maximizing $\left\langle Y_{22}\left(\theta^{\prime \prime}, \varphi^{\prime \prime}\right)+Y_{2-2}\left(\theta^{\prime \prime}, \varphi^{\prime \prime}\right)\right\rangle$ after having maximized $\left\langle Y_{20}\left(\theta^{\prime}, \varphi^{\prime}\right)\right\rangle$ (within the scope of the approximation mentioned above) is formally equivalent to minimizing the $\mathrm{POMT}_{1}$ given by eq 3 .

Equation 3 is at minimum for three scenarios. (a) $\beta_{\mathrm{VF}-\mathrm{OF}}=0^{\circ}$ : the main local ordering axis (i.e., the axis that orients preferentially parallel to the local director) is $\mathrm{Z}_{\mathrm{OF}}$. In this case, Z-ordering prevails. (b) $\beta_{\mathrm{VF}-\mathrm{OF}}=90^{\circ}$ and $\gamma_{\mathrm{VF}-\mathrm{OF}}=0^{\circ}$ : the main ordering axis is $\mathrm{X}_{\mathrm{OF}}$. In this case, $\mathrm{X}$-ordering prevails. (c) $\beta_{\mathrm{VF}-\mathrm{OF}}=90^{\circ}$ and $\gamma_{\mathrm{VF}-\mathrm{OF}}=90^{\circ}$ : the main ordering axis is $\mathrm{Y}_{\mathrm{OF}}$. In this case, Y-ordering prevails. ${ }^{38}$ As pointed out above, the definition of $(\mathrm{N}-\mathrm{H})_{\text {eff }}$ in mf-RDC implies Z-ordering. On the other hand, the standard tensorial approach is consistent with X-, Y-, or Z-ordering. We show below that this feature, along with rank 2 tensor eigenvalues and PAS, can provide important new information. 


\section{RESULTS AND DISCUSSION}

The Saupe tensor given by eq 8 is diagonalized using the Lapack computer program. ${ }^{39}$ This program registers the eigenvalues in ascending order of their magnitude, according to the convention $\left|S_{z z}\right|>\left|S_{y y}\right| \geq\left|S_{x x}\right|{ }^{40}$

3.1. $\mathbf{N}-\mathbf{H}$ Sites. 3.1.1. Standard Analysis. Ubiquitin comprises an $\alpha$-helix (residues 23-34), a $33_{10}$-helix (residues $56-59$ ), and a $\beta$-sheet with strands denoted $\beta_{1}-\beta_{5}$ (residues $2-7,12-17,41-45,49-50$, and $65-72$ ). A flexible end chain (residues 73-76) is also featured. Figure 3 shows the elements

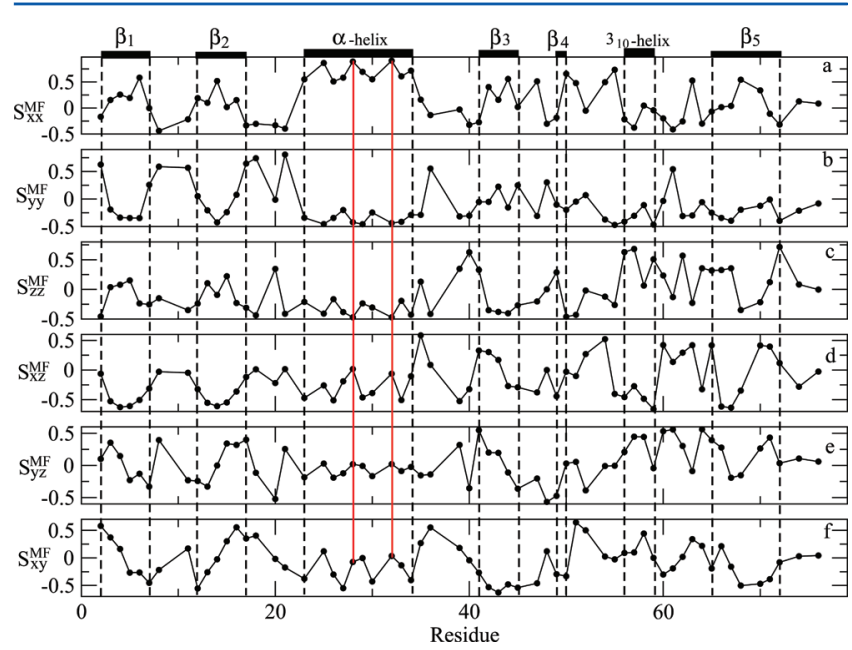

Figure 3. Elements of the (nondiagonal) local ordering Saupe tensor for the $\mathrm{N}-\mathrm{H}$ sites of ubiquitin. These data were derived from the $\left\langle Y_{2 m}\right\rangle$ 's of the tenth cycle of the SCRM analysis. ${ }^{4}$ The abscissa represents the protein sequence. The red vertical lines delineate the data corresponding to residues A28 and D32. On the basis of $S_{\text {rdc }}{ }^{2}$ errors of $1.3,6.2$, and $2.8 \%$ for the secondary structure elements, the loops, and the entire protein backbone, respectively, reported in ref 4 , we estimate the errors in the data shown to be approximately $5 \%$.

of the Saupe tensor given in the local MF frame described in Section 2.2.2. The secondary structure of ubiquitin is depicted on the top. The $\left\langle Y_{2 m}\right\rangle$ 's underlying Figure 3 correspond to the tenth cycle of the Self-Consistent RDC-based Model-free analysis (SCRM), ${ }^{4}$ applied to the structure of ubiquitin with PDB code 1UBI.

The elements of the (nondiagonal) Saupe tensors in the MF frame vary substantially along the protein backbone. For residue A28 (D32), depicted by the red vertical lines in Figure 3, the nondiagonal elements are $S_{x z}^{\mathrm{MF}}=-0.074(0.035), S_{y z}^{\mathrm{MF}}=0.019$ $(0.020)$, and $S_{x y}^{\mathrm{MF}}=0.016(-0.063)$. These values are very small, indicating that the respective Saupe tensors are nearly diagonal in MF. The diagonal elements are $S_{x x}^{\mathrm{MF}}=0.893(0.909)$, $S_{y y}^{\mathrm{MF}}=-0.422(-0.437)$, and $S_{z z}^{\mathrm{MF}}=-0.471(-0.472)$, for residue $\mathrm{A} 28(\mathrm{D} 32)$. The largest positive order parameter is $S_{x x}^{\mathrm{MF}}$, indicating that $\mathrm{X}_{\mathrm{MF}}$, i.e., the orientation perpendicular to the $\mathrm{N}-\mathrm{H}$ bond in the peptide-bond plane, is the main local ordering axis for these residues.

The eigenvalues of $\boldsymbol{S}_{1}$ obtained by Lapack diagonalization are shown in Figure 4. The Saupe scheme order parameters are $S_{x x}=-0.481(-0.481), S_{y y}=-0.418(-0.427)$, and $S_{z z}=0.899$ $(0.903)$, and the rhombic Wigner scheme order parameter, $S_{2}^{2}$, is $-0.047(-0.042)$ for residue A28 (D32). As expected based on Figure 3, and the convention $\left|S_{z z}\right|>\left|S_{y y}\right| \geq\left|S_{x x}\right|$ used by the Lapack program, ${ }^{39}$ within a good approximation, the
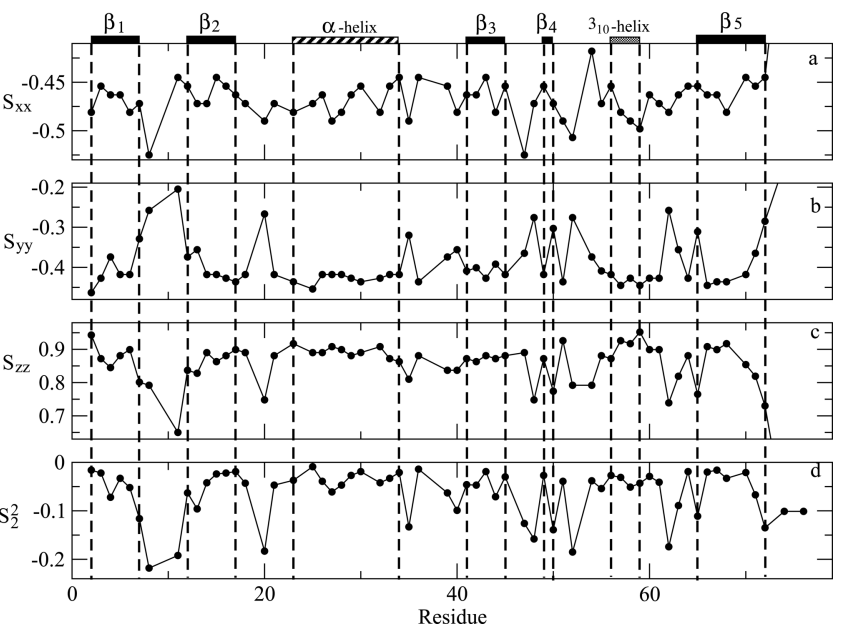

Figure 4. Saupe scheme order parameters, $S_{x x}, S_{y y}$, and $S_{z z}$ and the rhombic Wigner scheme order parameter, $S_{2}^{2}$, obtained by subjecting the tensors shown in Figure 3 to Lapack diagonalization. The abscissa represents the protein sequence. We estimate the errors to be approximately 5\%, according to ref 4 .

diagonalization process exchanged $\mathrm{X}_{\mathrm{MF}}$ and $\mathrm{Z}_{\mathrm{MF}}$, which became $\mathrm{Z}_{\mathrm{OF}}$ and $\mathrm{X}_{\mathrm{OF}}$, respectively. This agrees with the values of the Euler angles shown in Figure 5, obtained by a standard

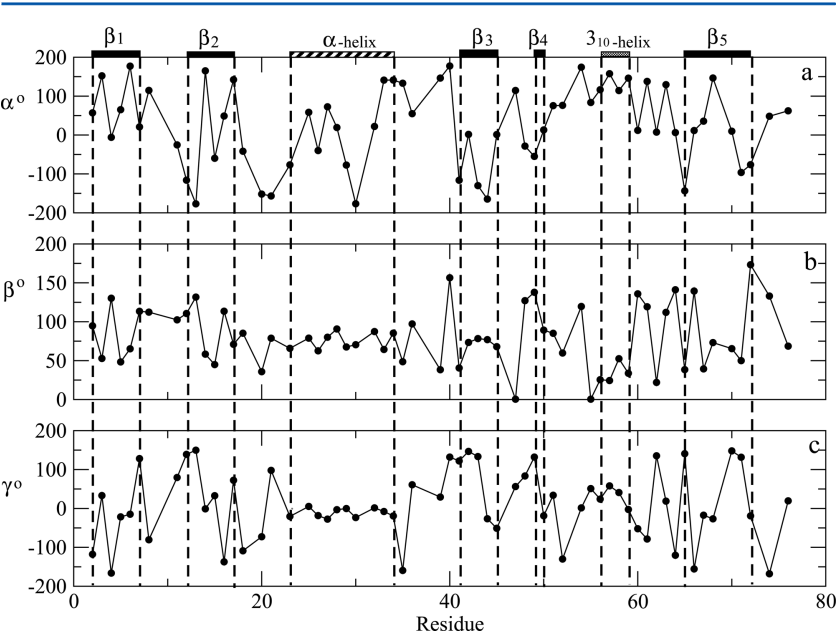

Figure 5. Euler angles of the Wigner rotations that generated the order parameters shown in Figure 4. The abscissa represents the protein sequence. We estimate the errors to be approximately $5 \%$, according to ref 4

counter-clockwise ZYZ Wigner rotation. These values are $\alpha=$ $19.3^{\circ}\left(22.1^{\circ}\right), \beta=90.7^{\circ}\left(87.4^{\circ}\right)$, and $\gamma=-3.2^{\circ}\left(1.5^{\circ}\right)$ for residue $\mathrm{A} 28$ (D32). The $\alpha$ angle represents a small rotation around $\mathrm{Z}_{\mathrm{MF}}$. The $\beta$ angle represents a $90^{\circ}$ rotation around the new $Y$-axis. The $\gamma$ angle is nearly zero given that the OF frame is nearly axially symmetric for these residues, as shown by the small corresponding $S_{2}^{2}$ values (Figure $4 \mathrm{~d}$ ).

A plausible assignment for $\mathrm{Z}_{\mathrm{OF}}$, which is perpendicular to $\mathrm{X}_{\mathrm{OF}}=\mathrm{N}-\mathrm{H}$, is the $\mathrm{C}_{i-1}^{\alpha}-\mathrm{C}_{i}^{\alpha}$ axis. The standard tilt angle between $\mathrm{C}_{i-1}^{\alpha}-\mathrm{C}_{i}^{\alpha}$ and $\mathrm{N}-\mathrm{H}$ (which points along $\mathrm{Z}_{\mathrm{DF}}$ ) is $-101.3^{\circ}$ (refs 41-43). One may either adopt the orthoGaussian Axial Fluctuations (GAF) model of ref 41, where $\beta_{\mathrm{OF}-\mathrm{DF}}=90^{\circ}$, or assign $\mathrm{Z}_{\mathrm{OF}}$ as an approximation to the $\mathrm{C}_{i-1}^{\alpha}-$ $C_{i}^{\alpha}$ axis. There is compelling evidence for $C_{i-1}^{\alpha}-C_{i}^{\alpha}$ serving as the main ordering axis at $\mathrm{N}-\mathrm{H}$ sites in proteins. We found 
with SRLS-based fitting of ${ }^{15} \mathrm{~N}$ spin relaxation data that the angle between $\mathrm{Z}_{\mathrm{OF}}$ and $\mathrm{Z}_{\mathrm{DF}}$ is within $1-2^{\circ}$ from $-101.3^{\circ}$ (refs 33 and 34). $C_{i-1}^{\alpha}-C_{i}^{\alpha}$ as main ordering axis is encoded in the $3 D$ GAF model ${ }^{42,43}$ which has been used extensively, including the analysis of ${ }^{15} \mathrm{~N}-{ }^{1} \mathrm{H}$ RDCs in proteins. ${ }^{44,45}$ Molecular dynamics studies found that crankshaft backbone motions, centered at the $C_{i-1}^{\alpha}-C_{i}^{\alpha}$ axis, occur pervasively in proteins. ${ }^{46-49}$

We assign $\mathrm{Z}_{\mathrm{OF}}$ as $\mathrm{C}_{i-1}^{\alpha}-\mathrm{C}_{i}^{\alpha}$ for residues A28 and D32 of the $\alpha$-helix. For all of the $\mathrm{N}-\mathrm{H}$ sites within the $\alpha$-helix, the $\beta$ angle is close to $90^{\circ}$ (Figure $5 \mathrm{~b}$ ), and the $\gamma$ angle is close to $0^{\circ}$ (Figure $5 \mathrm{c}$ ), in agreement with the geometric arrangement characteristic of residues A28 and D32. On the basis of this, and on the similarity between corresponding Saupe tensor eigenvalues (Figure 4; the apparently large variations in Figure $4 \mathrm{a}$ are due to an expanded scale), we assign $\mathrm{Z}_{\mathrm{OF}}$ as $\mathrm{C}_{i-1}^{\alpha}-\mathrm{C}_{i}^{\alpha}$ for the entire $\alpha$-helix. For the remaining $\mathrm{N}-\mathrm{H}$ sites of ubiquitin, the assignment of $\mathrm{Z}_{\mathrm{OF}}$ (in general, of the entire PAS of $S_{1}$ ) has to await the development of the general scheme for RDC analysis delineated in Section 2.1.

The finding that for the $\alpha$-helix of ubiquitin the main ordering axis is $\mathrm{C}_{i-1}^{\alpha}-\mathrm{C}_{i}^{\alpha}$ (which constitutes insightful structural information) is due to the tensorial description of the local ordering. Additional information emerging from the standard analysis appears in the next section.

3.1.2. Comparison between the Standard Analysis and $m f-R D C$. The squared generalized order parameter, $S_{\mathrm{rdc}}^{2}$, is considered as a measure of the degree of local ordering. We compare it with $S_{0}^{2}$, which represents the strength of the local ordering in the tensorial perspective. These parameters are depicted in Figure 6a, with $S_{0}^{2}$ shown in blue and $S_{\text {rdc }}^{2}$ shown in
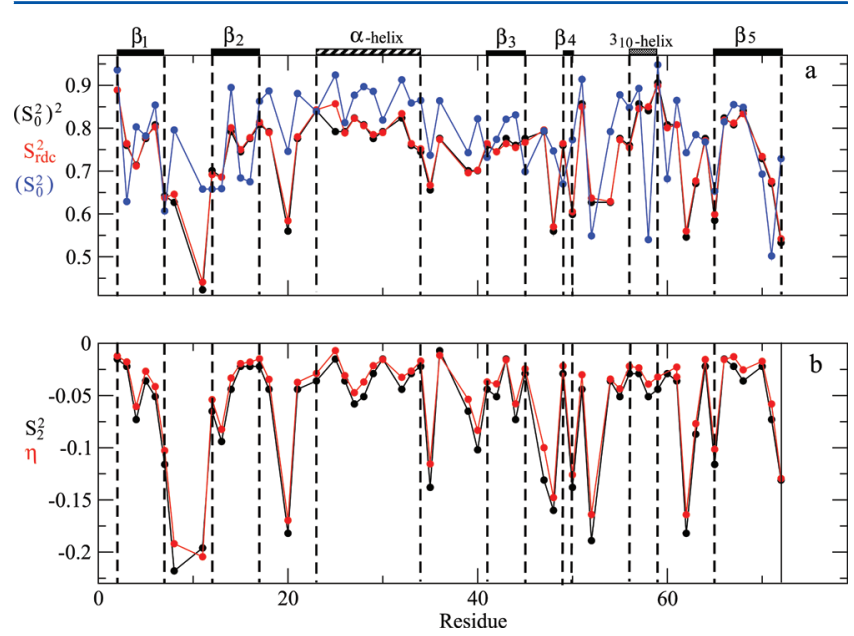

Figure 6. Axial Wigner scheme order parameter, $S_{0}^{2}$ (blue), its square $\left(S_{0}^{2}\right)^{2}$ (black), and squared generalized order parameter, $S_{\mathrm{rdc}}{ }^{2}$ (red) for the $\mathrm{N}-\mathrm{H}$ sites of ubiquitin (a). Rhombic Wigner scheme order parameter, $S_{2}^{2}$ (black), and amplitude of anisotropic disorder, $\eta$ (calculated according to eq 10 and depicted with reversed sign) (red), for the $\mathrm{N}-\mathrm{H}$ sites of ubiquitin (b). The abscissa represents the protein sequence. We estimate the errors to be approximately $5 \%$, according to ref 4 .

red. It can be seen that the respective patterns differ. $S_{0}^{2}$ and $S_{\text {rdc }}^{2}$ are related numerically as follows. The original definition of $S_{\mathrm{rdc}}{ }^{2}$ is given by eq 9. ${ }^{5}$ An equivalent definition is

$$
S_{\mathrm{rdc}}^{2}=\sum_{m=0, \pm 1, \pm 2}\left|\left\langle D_{0 m}^{2}\right\rangle\left\langle D_{0-m}^{2}\right\rangle\right|
$$

$S_{\text {rdc }}{ }^{2}$ is the same in any frame. In the PAS of the ordering tensor, where only $\left\langle D_{00}^{2}\right\rangle$ and $\left\langle D_{02}^{2}+D_{0-2}^{2}\right\rangle$ survive, one has

$$
S_{\mathrm{rdc}}^{2}=\left\langle D_{00}^{2}\right\rangle^{2}+2\left\{\operatorname{Re}\left\langle D_{02}^{2}\right\rangle\right\}^{2}
$$

Given that $S_{0}^{2}=\left\langle D_{0}^{2}\right\rangle$ and $S_{2}^{2}=\left\langle D_{02}^{2}+D_{0-2}^{2}\right\rangle=2\left\{\operatorname{Re}\left\langle D_{02}^{2}\right\rangle\right\}$, one has

$$
S_{\mathrm{rdc}}^{2}=\left(S_{0}^{2}\right)^{2}+\frac{1}{2}\left(S_{2}^{2}\right)^{2}
$$

$S_{0}^{2}$ and $S_{2}^{2}$ are independent eigenvalues of the local ordering tensor, which represnt different physical properties. $S_{\text {rdc }}{ }^{2}$ is a parameter that comprises information on both. When the rhombic contribution to $S_{\mathrm{rdc}}{ }^{2}$ is relatively small, one has $S_{\mathrm{rdc}}{ }^{2} \sim$ $\left(S_{0}^{2}\right)^{2}$; this represents the axial ordering scenario. To evaluate the deviation from axiality, we show $\left(S_{0}^{2}\right)^{2}$ in Figure 6a (black). It can be seen that $S_{\text {rdc }}^{2}$ and $\left(S_{0}^{2}\right)^{2}$ are very close to one another, indicating that the rhombic term in eq 17 makes a small contribution to $S_{\mathrm{rdc}}{ }^{2}$. An expression equivalent to the one depicted in eq 17 appears in ref 14 . However, to our knowledge, its utility in the context mentioned above was not pointed out previously.

The parameter $\eta$ represents the anisotropy of the local disorder. We show $\eta$ (calculated according to eq 10) and its formal analogue $S_{2}^{2}$, which represents the rhombicity of the local ordering tensor, in Figure $6 \mathrm{~b}$. The parameter $\eta$ is by definition positive. Since based on the convention $\left|S_{z z}\right|>\left|S_{y y}\right| \geq\left|S_{x x}\right| S_{2}^{2}$ is negative, we show $\eta$ with reversed sign to render the comparison convenient. Note that when the Euler angles $\Omega_{\mathrm{OF}-\mathrm{DF}}$ will be determined, the sign of $S_{2}^{2}$ will provide physically insightful information.

Figure $6 \mathrm{~b}$ shows that $\eta$ is smaller in absolute value than $S_{2}^{2}$, which is itself small (the maximum value of $S_{2}^{2}$ is $(3 / 2)^{1 / 2}$ ). Thus, the anisotropic disorder is small at the $\mathrm{N}-\mathrm{H}$ sites of ubiquitin. These important assessments, including the quantitative evaluation of the deviation of $S_{\mathrm{rdc}}^{2}$ from $\left(S_{0}^{2}\right)^{2}$, require taking cognizance of the fact that $S_{\mathrm{rdc}}{ }^{2}$ is given by eq 17; $\eta$ is given by $\left[\left(0.5 \times\left(S_{2}^{2}\right)^{2}\right) /\left(\left(S_{0}^{2}\right)^{2}+0.5 \times\left(S_{2}^{2}\right)^{2}\right)\right]^{1 / 2}($ eqs 10 and 17); and $S_{0}^{2}$ and $S_{2}^{2}$ can be derived from the mf-RDC parameters (eq 13). Figure 7 shows $\varphi_{\text {eff }} \theta_{\text {eff, }}$ and $\bar{\Phi}^{\prime} . \Delta \theta_{\text {eff }}$ and $\Delta \varphi_{\text {eff }}$

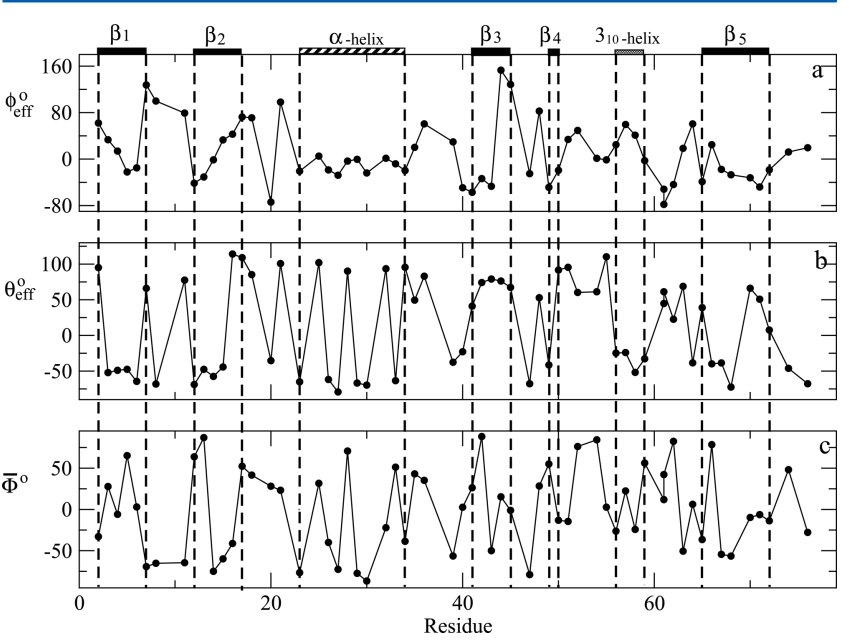

Figure 7. mf-RDC parameters $\varphi_{\text {eff }}(\mathrm{a}), \theta_{\text {eff }}(\mathrm{b})$, and $\bar{\Phi}^{\prime}(\mathrm{c})$, for the $\mathrm{N}-\mathrm{H}$ sites of ubiquitin, derived from the $\left\langle Y_{2 m}\right\rangle$ 's of the tenth cycle of the SCRM analysis. ${ }^{4}$ The abscissa represents the protein sequence.

represent the difference in the orientation of $(\mathrm{N}-\mathrm{H})_{\text {eff }}$ and $(\mathrm{N}-\mathrm{H})_{\text {static }}$ in the protein structure. These quantities are on the order of several degrees (Figure 4 of ref 10), indicating that $(\mathrm{N}-\mathrm{H})_{\text {eff }}$ and $(\mathrm{N}-\mathrm{H})_{\text {static }}$ are close to one another. One may 
conclude that $(\mathrm{N}-\mathrm{H})_{\mathrm{av}}$ and $(\mathrm{N}-\mathrm{H})_{\text {static }}$ are also close to one another.

3.2. $\mathbf{C}-\mathrm{C}_{\text {methyl }}$ Sites. 3 3.2.1. Standard Analysis. Figure 8 (Figure 9) shows the Saupe tensor elements in MF for the
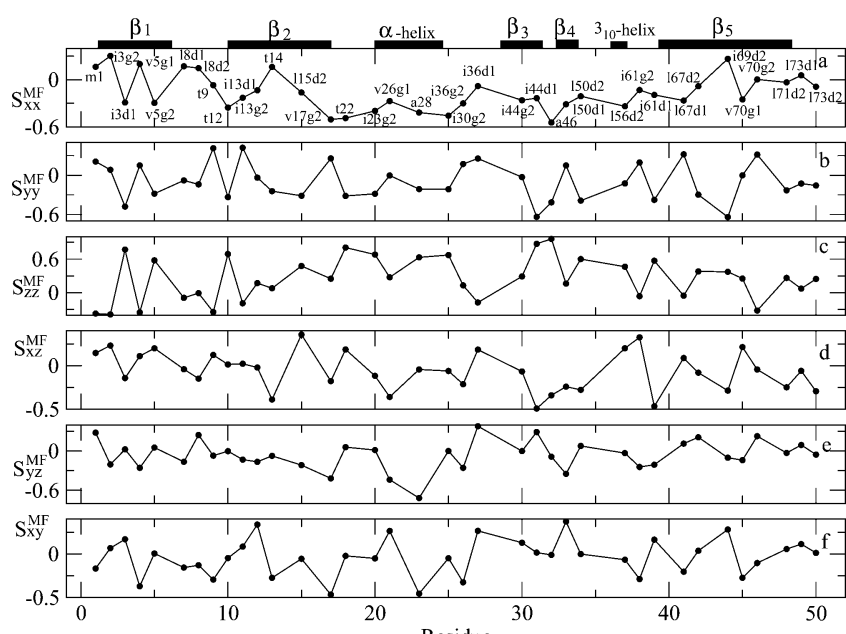

Figure 8. Elements of the local ordering Saupe tensor for the $\mathrm{C}-\mathrm{C}_{\text {methyl }}$ sites of ubiquitin derived from the $\left\langle Y_{2 m}\right\rangle$ 's of ref 13 , presented as a function of the protein sequence. Errors as in ref 13.

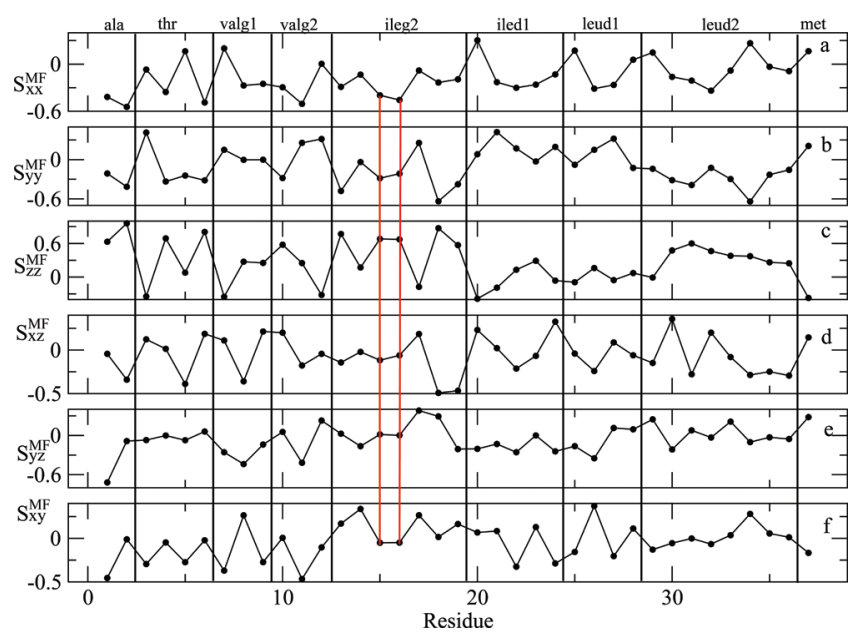

Figure 9. Elements of the local ordering Saupe tensor for the $\mathrm{C}-\mathrm{C}_{\text {methyl }}$ sites of ubiquitin derived from the $\left\langle Y_{2 m}\right\rangle$ 's of ref 13, presented as a function of the methyl type. The red vertical lines delineate the data corresponding to residues $\mathrm{I} \gamma_{2} 23$ and $\mathrm{I} \gamma_{2} 30$. Errors as in ref 13 .

$\mathrm{C}-\mathrm{C}_{\text {methyl }}$ sites of ubiquitin outlined in Section 2.2.2., depicted as a function of the protein sequence (methyl type). The (nondiagonal) Saupe tensor elements vary substantially in both representations. In Figure 9, one can distinguish a certain degree of regularity in the $S_{x x}$ and $S_{y z}$ values of Ile $\delta_{1}$ and the $S_{z z}$ and $S_{x y}$ values of the Leu $\delta_{2}$. In quite a few cases, $S_{z z}$ is positive and large. Figures 10 and 11 show the results of Lapack diagonalization applied to the data shown in Figure 9. The Saupe scheme order parameters $S_{x x}, S_{y y}$, and $S_{z z}$ and the Wigner scheme order parameter $S_{2}^{2}$ are shown in Figure 10. The Euler angles associated with the Wigner rotation from the MF frame to the PAS of $S_{1}$ are shown in Figure 11 .

Let us consider the $\mathrm{C}-\mathrm{C}_{\text {methyl }}$ sites of residues $\mathrm{I} \gamma_{2} 23$ and $\mathrm{I} \gamma_{2} 30$, depicted by the red vertical lines in Figure 9. The nondiagonal elements of the Saupe tensor given in MF are

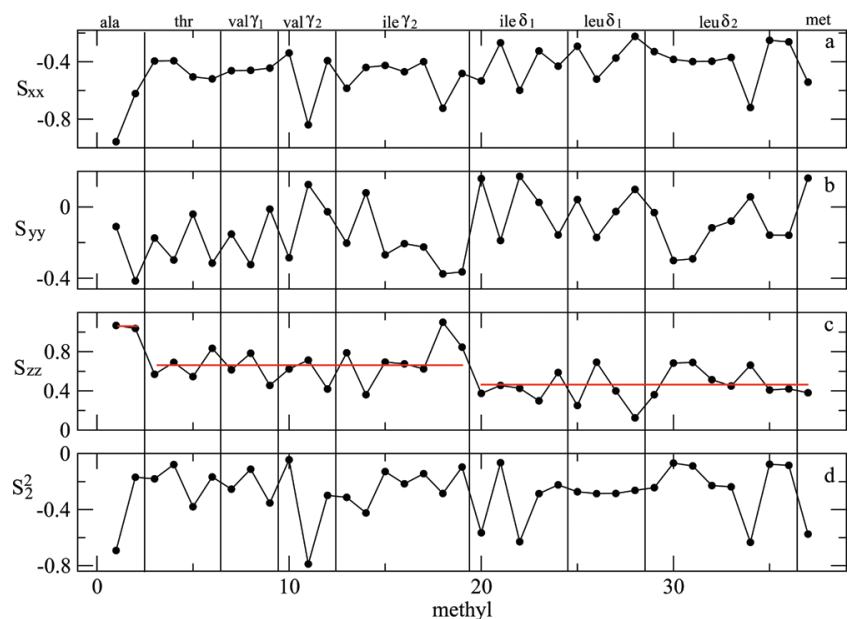

Figure 10. Saupe scheme order parameters, $S_{x x}, S_{y y}$, and $S_{z z}$ and the rhombic Wigner scheme order parameter, $S_{2}^{2}$, as a function of methyl type, obtained by subjecting the tensors shown in Figure 9 to Lapack diagonalization. The red lines represent averages over the residues encompassed.

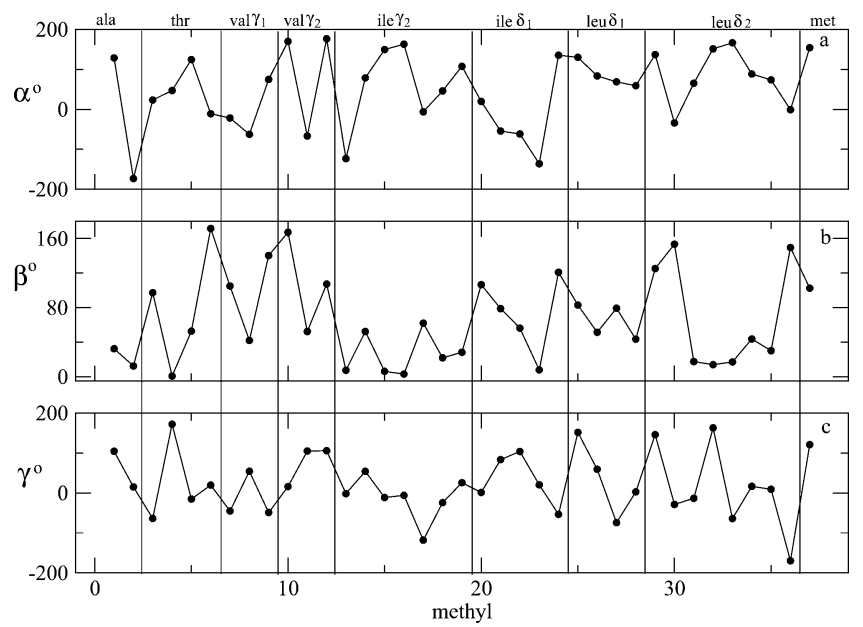

Figure 11. Euler angles of the Wigner rotations that generated the order parameters shown in Figure 10, as a function of methyl type.

$S_{x z}^{\mathrm{MF}}=-0.117(-0.061), S_{y z}^{\mathrm{MF}}=0.016(0.002)$, and $S_{x y}^{\mathrm{MF}}=0.051$ $(-0.049)$ for residue $\mathrm{I} \gamma_{2} 23\left(\mathrm{I} \gamma_{2} 30\right)$. These are small values, indicating that the respective Saupe tensors are nearly diagonal in MF. The diagonal elements are $S_{x x}^{\mathrm{MF}}=-0.397(-0.457)$, $S_{y y}^{\mathrm{MF}}=-0.285(-0.215)$, and $S_{z z}^{\mathrm{MF}}=0.681(0.672)$ for residue $\mathrm{I} \gamma_{2} 23\left(\mathrm{I} \gamma_{2} 30\right)$. The largest positive diagonal element is $S_{z z}$ indicating that $\mathrm{Z}_{\mathrm{MF}}$, i.e., the orientation pointing along the $\mathrm{C}-\mathrm{C}_{\text {methyl }}$ axis, is the main local ordering axis for these residues.

The eigenvalues of $S_{1}$ are $S_{x x}=-0.426(-0.470), S_{y y}=-0.268$ $(-0.206)$, and $S_{z z}=0.694(0.676)$ for residue $\mathrm{I} \gamma_{2} 23\left(\mathrm{I} \gamma_{2} 30\right)$ (Figure 10). The absolute values of these parameters are similar to their counterparts in the MF frame (Figure 9), and their designations are preserved. The diagonalization process has merely set equal to zero the small nondiagonal elements shown in Figure 9, updating accordingly the diagonal elements. This is supported by the Euler angles shown in Figure 11, which are $\alpha=149.7^{\circ}\left(163.1^{\circ}\right), \beta=6.3^{\circ}\left(3.1^{\circ}\right)$, and $\gamma=11.3^{\circ}\left(-6.0^{\circ}\right)$ for residue $\mathrm{I} \gamma_{2} 23\left(\mathrm{I} \gamma_{2} 30\right)$. Within a reasonably good approximation, this represents a $180^{\circ}$ rotation around $\mathrm{Z}_{\mathrm{MF}}$, to which the local 
ordering is invariant. Thus, for $\mathrm{C}-\mathrm{C}_{\text {methyl }}$ sites, the tensorial approach supports Z-ordering.

The rhombic order parameter, $S_{2}^{2}$, is $-0.129(-0.216)$ for residue $\mathrm{I} \gamma_{2} 23\left(\mathrm{I} \gamma_{2} 30\right)$. The extent of rhombicity is considerable.

For some of the $\mathrm{C}-\mathrm{C}_{\text {methyl }}$ sites of ubiquitin $\mathrm{S}_{z z}$ is larger than 0.5 in Figure 9, and the nondiagonal elements are relatively small. For these methyl groups, the $\mathrm{C}-\mathrm{C}_{\text {methyl }}$ axis is likely to be the main local ordering axis. For most $\mathrm{C}-\mathrm{C}_{\text {methyl }}$ sites, $S_{x x}$ is negative and close to -0.35 . $S_{y y}$ is negative for $76 \%$ of the C$\mathrm{C}_{\text {methyl }}$ sites. Figure 11 shows that nearly $50 \%$ of the $\mathrm{C}-\mathrm{C}_{\text {methyl }}$ sites have $\beta=20^{\circ} \pm 15^{\circ} ; 71 \%$ of the Ile $\gamma_{2} \mathrm{C}-\mathrm{C}_{\text {methyl }}$ sites have $\beta$ in the $5^{\circ}-20^{\circ}$ range; and $43 \%$ of the Leu $\delta_{2} \mathrm{C}-\mathrm{C}_{\text {methyl }}$ sites have $\beta$ in the $14^{\circ}-44^{\circ}$ range. $49 \%$ of all the $\mathrm{C}-\mathrm{C}_{\text {methyl }}$ sites, $71 \%$ of the $\mathrm{C}-\mathrm{C}_{\text {methyl }}$ sites of $\mathrm{Ile} \gamma_{2}$, and $62.5 \%$ of the $\mathrm{C}-\mathrm{C}_{\text {methyl }}$ sites of Leu $\delta_{2}$ have $\gamma$ less than $30^{\circ}$. Thus, in general the symmetry of the $S_{1}$ tensors for $\mathrm{I} \gamma_{2} 23$ and $\mathrm{I} \gamma_{2} 30$ is, within a reasonably good approximation, representative of the symmetry of the $S_{1}$ tensors for all of the $\mathrm{C}-\mathrm{C}_{\text {methyl }}$ sites of ubiquitin. One may conclude that $\mathrm{Z}_{\mathrm{OF}}$ resides within a solid angle centered at the $\mathrm{C}-\mathrm{C}_{\text {methyl }}$ axis; $\mathrm{X}_{\mathrm{OF}}$ resides within a solid angle centered at the orientation perpendicular to the $\mathrm{C}-\mathrm{C}_{\text {methyl }}$ axis in the plane defined by the $\mathrm{C}_{i-1}-\mathrm{C}_{i}$ bond and the $\mathrm{C}-\mathrm{C}_{\text {methyl }}$ axis; and $\mathrm{Y}_{\mathrm{OF}}$ is approximately perpendicular to this plane. Accurate assignment of the PAS of $S_{1}$ based solely on a tensorial analysis, which conveys important structural/ geometric information, has to await the development of the scheme outlined in Section 2.1. A quantitative treatment of the local ordering at the $\mathrm{C}-\mathrm{C}_{\text {methyl }}$ sites of ubiquitin, based on mf-RDC combined with additional independent methods of analysis, is presented in ref 13 .

The order parameter $S_{z z}$ is insightful. The red lines in Figure 10c show that $S_{z z}$ is $0.97,0.67$, and 0.47 for the methyl groups of alanine, threonine/valine/Ile $\gamma_{2}$ and Ile $\delta_{1} /$ leucine/methionine, respectively. We studied previously with SRLS ${ }^{2} \mathrm{H}$ spin relaxation from ${ }^{13} \mathrm{CDH}_{2}$ methyl groups of $\mathrm{Ca}^{2+}$-calmodulin in complex with the peptide smMLCKp, to find that $\left|c_{2}^{2}\right|$ (eq 3 ) also clusters into three groups. ${ }^{50}$ In that case, large, small, and medium $\left|c_{2}^{2}\right|$ values were found to correspond to alanine, methionine, and the remaining methyl types, respectively. Thus, while (substantial) ordering rhombicity (evaluated in terms of $\left.\left|c_{2}^{2}\right|\right)$ correlates with methyl-type/side-chain length in the presence of picosecond-nanosecond motions, ordering strength (evaluated in terms of $S_{0}^{2}$ ) correlates with methyl type/ side-chain length in the presence of picosecond-millisecond motions (note that $c_{0}^{2}$ and $S_{0}^{2}$, on the one hand, and $c_{0}^{2}$ and $S_{2}^{2}$, on the other hand, contain similar information). The overall effect of the large number of different dynamic processes occurring on the nanosecond-millisecond time scale is to render the effective ordering more symmetric but still sensitive to methyl type/side-chain length.

Figure $3 \mathrm{~b}$ of ref 13 shows that for the methyl groups associated with long side chains $\left(\mathrm{C}-\mathrm{C}_{\text {methyl }}\right)_{\text {eff }}$ deviates substantially from $\left(\mathrm{C}-\mathrm{C}_{\text {methyl }}\right)_{\text {static }}$. In such cases, it is important to allow for arbitrary orientation of $\left(\mathrm{C}-\mathrm{C}_{\text {methyl }}\right)_{\mathrm{av}}$ in the protein and determine this important geometric feature with data fitting. This can be accomplished with the comprehensive scheme outlined in Section 2.1.

3.2.2. Comparison between Saupe Tensor Diagonalization and $m f-R D C$. Figure 12 shows the parameters $S_{\mathrm{rdc}}{ }^{2}, \eta$, and $\bar{\Phi}^{\prime}$, and Figure 13 shows the angles $\theta_{\text {eff }}$ and $\varphi_{\text {eff }}$. These data have been reproduced from ref 13 . As shown by the red lines in Figure 12a, $S_{\text {rdc }}{ }^{2}$ distinguishes between the same three groups of methyl types as $S_{0}^{2}$ (see red lines in Figure 10c). However, the

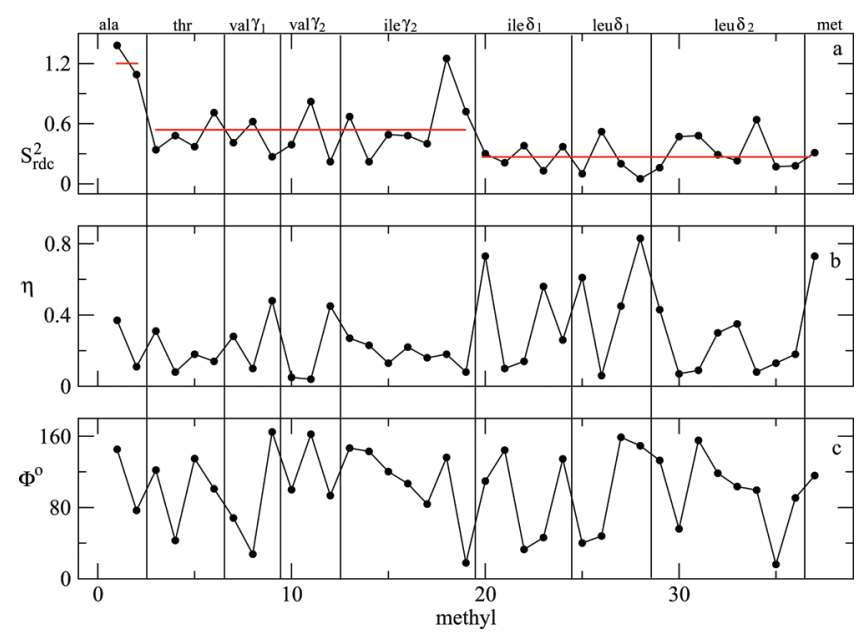

Figure 12. Squared generalized order parameter, $S_{\text {rdc }}^{2}$ (a); amplitude of anisotropic disorder, $\eta(\mathrm{b})$; and direction of anisotropic disorder, $\bar{\Phi}^{\prime}$ (c), for the $\mathrm{C}-\mathrm{C}_{\text {methyl }}$ sites of ubiquitin, as a function of methyl type, reproduced from ref 13 . The red lines represent averages over the residues encompassed.

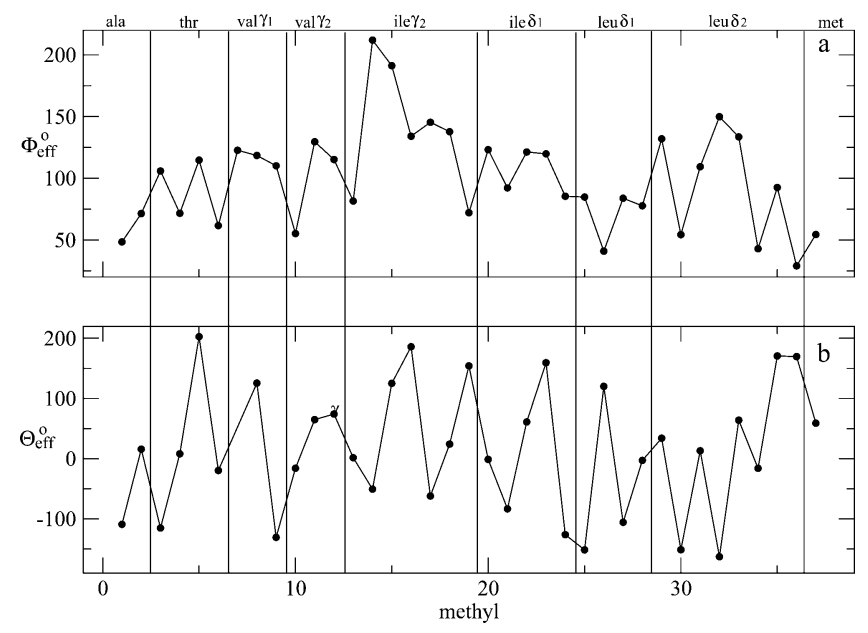

Figure 13. mf-RDC parameters $\varphi_{\text {eff }}(\mathrm{a})$ and $\theta_{\text {eff }}(\mathrm{b})$ for the C$\mathrm{C}_{\text {methyl }}$ sites of ubiquitin, as a function of methyl type, reproduced from ref 13 .

$S_{\mathrm{rdc}}{ }^{2}$ and $\left(S_{0}^{2}\right)^{2}$ patterns differ, and the corresponding absolute values differ, as shown in Figure 14a, where $S_{0}^{2}$ (blue) and $S_{\text {rdc }}^{2}$ (red) are superimposed. $\left(S_{0}^{2}\right)^{2}$ is also shown in Figure 14a (black). $S_{\text {rdc }}{ }^{2}$ and $\left(S_{0}^{2}\right)^{2}$ do not differ substantially, although as shown below, the local ordering at methyl sites is substantially rhombic.

In Figure 14b, we show $S_{2}^{2}$ superimposed on $\eta$ (taken from Table S3 of ref 13 and depicted with reversed sign). The differences are substantial, and the absolute values are considerable. Thus, for the $\mathrm{C}-\mathrm{C}_{\text {methyl }}$ sites of ubiquitin we detect relatively large rhombicity. $S_{0}^{2}$ and $S_{2}^{2}$ are distinctive measures of ordering strength and rhombicity. As pointed out above, $S_{\text {rdc }}^{2}$ and $\eta$ are composites of these properties. Therefore, it is useful to characterize the local ordering at the $\mathrm{C}-\mathrm{C}_{\text {methyl }}$ sites of ubiquitin in terms of $S_{x x}, S_{y y}$, and $S_{z z}$ or $S_{0}^{2}$ and $S_{2}^{2}$, and the PAS of $\boldsymbol{S}_{\mathbf{l}}$.

3.2.3. Comparison between Saupe Tensor Diagonalization and ${ }^{15} \mathrm{~N}$ Spin Relaxation. We studied previously ${ }^{2} \mathrm{H}$ spin relaxation from ${ }^{13} \mathrm{CDH}_{2}$ methyl groups in ubiquitin with the 


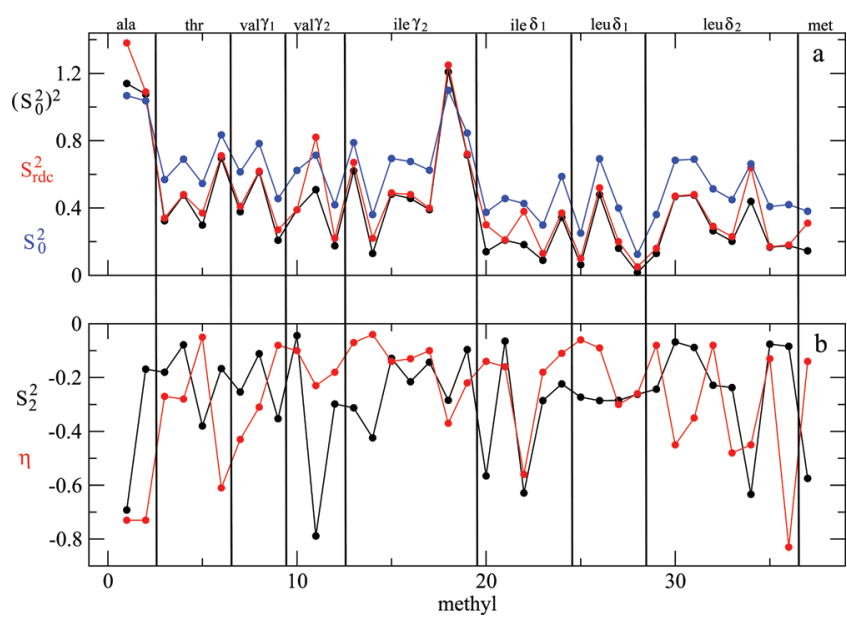

Figure 14. Axial Wigner scheme order parameter, $S_{0}^{2}$ (blue); squared axial Wigner scheme order parameter, $\left(S_{0}^{2}\right)^{2}$ (black); squared generalized order parameter, $S_{\text {rdc }}^{2}$ (red) (a). Rhombic Wigner scheme order parameter, $S_{2}^{2}$ (black); amplitude of anisotropic disorder, $\eta$, taken from Table S3 of ref 13 and depicted with reversed sign (red) (b). $S_{0}^{2}$ and $S_{2}^{2}$ are the same as $S_{z z}$ and $S_{2}^{2}$ in Figure $10 . S_{\text {rdc }}^{2}$ is taken from Figure 12a. The abscissa represents methyl type.

SRLS approach. ${ }^{51}$ Similar to the methyl groups of $\mathrm{Ca}^{2+}$ calmodulin*smMLCKp, ${ }^{50}$ the methyl groups of ubiquitin also exhibit large, small, and medium rhombicity evaluated by $\left|c_{2}^{2}\right|$ for alanine, methionine, and other methyl types, respectively. In the spin relaxation studies, the angle $\beta_{\mathrm{OF}-\mathrm{QF}}$ was allowed to vary. In the present analysis, the angle $\beta_{\mathrm{OF}-\mathrm{QF}}$ is implicitly set equal to its standard value of $110.5^{\circ}$ (ref 52). It is of interest to compare the parameters $S_{0}^{2}$ and $S_{2}^{2}$ obtained from spin relaxation, which probes the picosecond-nanosecond regime, with their counterparts obtained from RDCs, which probe the picosecond-millisecond regime. To render the comparison

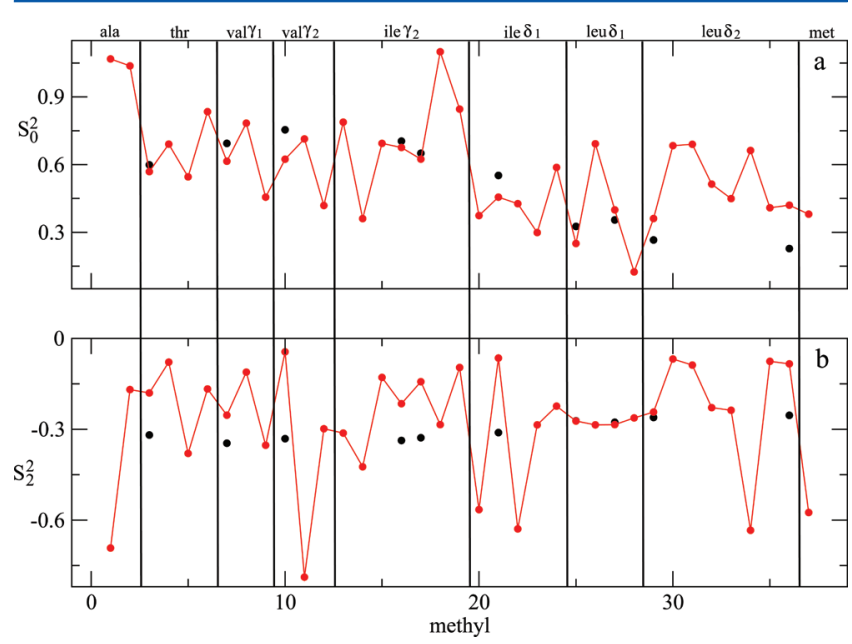

Figure 15. $S_{0}^{2}$ obtained with SRLS analysis of ${ }^{2} \mathrm{H}$ spin relaxation from ${ }^{13} \mathrm{CDH}_{2}$ methyl groups of ubiquitin, reproduced from ref 49 (black). $S_{0}^{2}$ obtained in this study with the standard tensorial approach for the $\mathrm{C}-\mathrm{C}_{\text {methyl }}$ sites of ubiquitin (red) (a). $S_{2}^{2}$ obtained with SRLS analysis of ${ }^{2} \mathrm{H}$ spin relaxation from ${ }^{13} \mathrm{CDH}_{2}$ methyl groups of ubiquitin, reproduced from ref 49 (black). $S_{2}^{2}$ obtained in this study with the standard tensorial approach for the $\mathrm{C}-\mathrm{C}_{\text {methyl }}$ sites of ubiquitin (red) (b).

meaningful, we show in Figure 15 in black only those data from ${ }^{2} \mathrm{H}$ relaxation that were obtained with $\beta_{\mathrm{OF}-\mathrm{QF}}$ virtually equal to $110.5^{\circ}$. The red symbols and lines represent the RDC-based $S_{0}^{2}$ and $S_{2}^{2}$ values obtained with the standard analysis.

As expected, the strength of the local ordering decreases in the presence of nanosecond-millisecond local motions (Figure 15a). For $75 \%$ of the cases, the difference in ordering strength is small. Figure $15 \mathrm{~b}$ shows $S_{2}^{2}$. It can be seen that the rhombicity of the local ordering is consistently smaller in the presence of nanosecond-millisecond motions. For 70\% of the cases, the reduction in this property is substantial. This indicates that the rhombic term of $S_{\mathrm{rdc}}^{2}$ (eq 17) dominates the difference between this parameter (ref 13) and its spinrelaxation-derived analogue (ref 49). The cases in which the rhombicity of the local ordering is virtually unaffected by nanosecond-millisecond motions include leucine methyl groups. For the same methyl groups, the strength of the local ordering is also nearly unaffected. Clearly, further studies are warranted to elucidate the effect of nanosecond-millisecond motions.

3.2.4. Comparison among Various Methods. The major approaches for treating experimental RDCs from proteins include $\mathrm{MFA}^{4,10}$ the method called Direct Interpretation of Dipolar Coupling (DIDC), ${ }^{14,17}$ and the method called Dynamic Meccano. ${ }^{16}$ We use eq 5 as measure, as this formulation comprises the maximum amount of information one can derive in principle from an appropriate set of experimental RDCs without scaling requirements.

Let us assume that mf-RDC is used within the scope of the MFA method. One would still like to have $\Omega_{\mathrm{MF}-\mathrm{VF}}=\left(\alpha_{\mathrm{MF}-\mathrm{VF}}\right.$, $\left.\beta_{\mathrm{MF}-\mathrm{VF}}, 0\right)$ and $\Omega_{\mathrm{OF}-\mathrm{DF}}=\left(\alpha_{\mathrm{OF}-\mathrm{DF}}, \beta_{\mathrm{OF}-\mathrm{DF}}, 0\right)$ as variables. In addition, one would like to eliminate the requirement for scaling the $\left\langle Y_{2 m}\right\rangle$ 's. In DIDC, ${ }^{14,17}$ both $S_{\mathrm{g}}$ and $\boldsymbol{S}_{1}$ are defined with respect to MF, which does not have to be known. In this case, in addition to forgoing the determination of $\Omega_{\mathrm{MF}-\mathrm{VF}}$ and $\Omega_{\mathrm{OF}-\mathrm{DF}}$, the determination of $\Omega_{\mathrm{AF}-\mathrm{MF}}$ is also forgone. The enhanced version of DIDC developed in ref 17 treats the local director in the same manner as mf-RDC.

In the Dynamic Meccano method, MF is not an independently known frame. Hence, $\Omega_{\mathrm{AF}-\mathrm{MF}}$ might be affected by fitting issues. The local director is given by the orientation of the peptide-bond plane in the context of MF. Thus, $\Omega_{\mathrm{MF}-\mathrm{VF}}$ is encoded in this method. The angles $\Omega_{\mathrm{OF}-\mathrm{DF}}$ are not allowed to vary.

\section{CONCLUSIONS}

$\mathrm{mf}-\mathrm{RDC}$ is one of the more elaborate methods for treating $\mathrm{RDCs}$ from flexible proteins. The global ordering is treated according to the standard tensorial procedure. The local ordering is treated with a specially designed method, mf-RDC. This approach yields scalar parameters that can be recast as the eigenvalues of the rank 2 local ordering tensor. On the other hand, the mf-RDC frame transformations are not the same as the conventional Wigner rotation. Here, we treat the local ordering with the standard tensorial approach that yields the eigenvalues and (through a conventional Wigner rotation) the PAS of the rank 2 local ordering tensor. New information on, hence new insights into, RDC-detected local ordering in proteins is obtained by virtue of the clear physical meaning and internal consistency of the tensorial representation. This includes assignment of main ordering axes, evaluation of the extent of local ordering rhombicity, and the detection of correlation between ordering strength/rhombicity and methyl type. Several important features, such as arbitrary orientation of the average spin-bearing bond in the protein, and arbitrary orientation of the local ordering frame in the probe, are not 
inherent to any of the current methods for treating RDCs from proteins. A scheme based on the tensorial perspective that accomplishes this is formulated.

\section{AUTHOR INFORMATION}

\section{Corresponding Author}

*E-mail: meirove@biu.ac.il. Phone: 972-3-531-8049. Fax: 9723-738-4058.

\section{Notes}

The authors declare no competing financial interest.

\section{ACKNOWLEDGMENTS}

E.M. thanks Prof. Zeev Luz for numerous inspiring and enlightening discussions. We gratefully acknowledge Prof. Antonino Polimeno and Dr. Mirco Zerbetto of the University of Padova, Italy, for giving us the computer program that diagonalizes the Saupe matrix, and for fruitful discussions. This work was supported by the Israel Science Foundation (Grant No. 347/07 to E.M.), the Binational Science Foundation (Grant No. 2006050 to E.M. and Jack H. Freed), the GermanIsraeli Science Foundation for Scientific Research and Development (Grant No. 928-190.0/2006 to E.M. and C.G.), and the Damadian Center for Magnetic Resonance at Bar-Ilan University, Israel. E.M. gratefully acknowledges the hospitality of the Department of Computational and Systems Biology, University of Pittsburgh School of Medicine, where she spent the summer of 2011, in the course of which this work was promoted. C.G. acknowledges support from the Max Planck Society and the European Research Council (ERC grant agreement no. 233227 to C.G.).

\section{REFERENCES}

(1) Tjandra, N; Bax, A. Science 1997, 278, 1111-1114.

(2) Tolman, J. R.; Flanagan, J. M.; Kennedy, M. A.; Prestegard, J. H. Nat. Struct. Biol. 1997, 4, 292-297.

(3) Blackledge, M. Prog. Nucl. Magn. Reson. Spectrosc. 2005, 46, $26-61$.

(4) Lakomek, N. A.; Walter, K. F. A.; Fares, C.; Lange, O. F.; de Groot, B. L.; Grübmuller, H.; Brüschweiler, R.; Munk, R. B. A.; Becker, S.; Meiler, J.; Griesinger, C. J. Biomol. NMR. 2008, 41, 139-155.

(5) Meiler, J; Prompers, J. J.; Peti, W.; Griesinger, C.; Brüschweiler, R. J. Am. Chem. Soc. 2001, 123, 6098-6107.

(6) Peti, W.; Meiler, J.; Brüschweiler, R.; Griesinger, C. J. Am. Chem. Soc. 2002, 124, 5822-5833.

(7) Hus, J.-C.; Peti, W.; Griesinger, C.; Bruschweiler, R. J. Am. Chem. Soc. 2003, 125, 5596-5597.

(8) Meiler, J.; Peti, W.; Griesinger, C. J. Am. Chem. Soc. 2003, 125, 8072-8073.

(9) Lakomek, N. A.; Fares, C.; Becker, S.; Carlomagno, T.; Meiler, J.; Griesinger, C. Angew. Chem., Int. Ed. 2005, 44, 7776-7778.

(10) Lakomek, N. A.; Carlomagno, T.; Becker, S.; Griesinger, C.; Meiler, J. J. Biomol. NMR 2006, 34, 101-115.

(11) Lange, O. F.; Lakomek, N. A.; Fares, C.; Schroder, G. F.; Walter, K. F. A.; Becker, S.; Meiler, J.; Grübmuller, H.; Griesinger, C. B.L. de Groot Science 2008, 320, 1471-1475.

(12) Jensen, M. R.; Markwick, P.; Meier, S.; Griesinger, C.; Zweckstetter., M.; Grzesiek, S.; Bernado, P.; Blackledge, M. Structure 2009, 17, 1169-1185.

(13) Fares, C.; Lakomek, N. A.; Walter, K. F. A.; Frank, B. T. C.; Meiler, J.; Becker, S.; Griesinger, C. J. Biomol. NMR 2009, 45, 23-44.

(14) Tolman, J. R. J. Am. Chem. Soc. 2002, 124, 12020-12030.

(15) Deschamps, M.; Campbell, I. D.; Boyd, J. J. Magn. Reson. 2005, $172,118-132$.
(16) Bouvignies, G.; Markwick, P.; Brüschweiler, R.; Blackledge, M. J. Am. Chem. Soc. 2006, 128, 15100-15101.

(17) Yao, L.; Vögeli, B.; Torchia, D. A.; Bax, A. J. Phys. Chem. B 2008, $112,6045-6056$.

(18) Salmon, L.; Bouvignies, G.; Markwick, P.; Lakomek, N.; Showalter, S.; Li, A.-W.; Walter, K.; Griesinger, C.; Brüschweiler, R.; Blackledge, M. Angew. Chem., Int. Ed. 2009, 48, 4154-4157.

(19) Guo, C.; Godoy-Ruiz, R.; Tugarinov, V. J. Am. Chem. Soc. 2010, 132, 13984-13987.

(20) Salmon, L.; Nodet, G.; Ozenne, V.; Yin, G.; Jensen, M. R; Zweckstetter, M.; Blackledge, M. J. Am. Chem. Soc. 2010, 132, 84078418.

(21) Salmon, L.; Bouvignies, G.; Marckwick, P.; Blackledge, M. Biochemistry 2011, 50, 5735.

(22) Fitzkee, N. C.; Bax, A. J. Biomol. NMR 2010, 48, 65-70.

(23) Sgourakis, N. G.; Lange, O. F.; DiMaio, F.; André, I.; Fitzkee, N. C.; Rossi, P.; Montelione, G. T.; Bax, A.; Baker, D. J. Am. Chem. Soc. 2011, 133, 6288-6298.

(24) Higman, V. A.; Boyd, J.; Smith, L. J.; Redfield, C. J. Biomol NMR 2011, 49, 53-60.

(25) Jarymowycz, V. A.; Stone, M. J. Chem. Rev. 2006, 106, 16241671.

(26) Palmer, A. G., III; Massi., F. Chem. Rev. 2006, 106, 17001719.

(27) NMR of Liquid Crystals; Emsley, J. W., Ed.; Reidel: Dordrecht, 1983.

(28) The Molecular Dynamics of Liquid Crystals; Luckhurst, G. R, Veracini, C. A., Eds.; Kluwer Academic Publishers: The Netherlands, 1994.

(29) Emsley, J. W.; Luckhurst, G. R. Mol. Phys. 1980, 41, 19-29.

(30) Almond, A.; Bunkenborg, J.; Franch, T.; Gotfredsen, H.; Duus, J. O. J. Am. Chem. Soc. 2001, 123, 4792-4802.

(31) Samulski, E. T.; Dong, R. Y. J. Chem. Phys. 1982, 77, 50905096.

(32) Polnaszek, C. F.; Freed, J. H. J. Phys. Chem. 1975, 79, 22832306.

(33) Meirovitch, E.; Shapiro, Yu. E.; Polimeno, A.; Freed, J. H. J. Phys. Chem. A 2006, 110, 8366-8396.

(34) Meirovitch, E.; Shapiro, Yu. E.; Polimeno, A.; Freed, J. H. Prog. NMR Spectrosc. 2010, 56, 360-405.

(35) Moltke, S.; Grzesiek, S. J. Biomol NMR 1999, 15, 77-82.

(36) Lipari, G.; Szabo, A. J. Am. Chem. Soc. 1982, 104, 45464559.

(37) Szabo, A. J. Chem. Phys. 1980, 72, 4620-4626.

(38) Barnes, J. P.; Freed, J. H. Biophys. J. 1998, 75, 2532-2546.

(39) MINPACK: numerical library for function minimization and leastsquares solutions, by Nelson H. F. Beebe Department of Mathematics, University of Utah; www.math.edu/software/minpack.html.

(40) Clore, G. M.; Gronenborn, A. M.; Bax, A. J. Magn. Reson. 1998, 133, 216-221.

(41) Bernado, P.; Blackledge, M. J. Am. Chem. Soc. 2004, 126, 49074920.

(42) Bremi, T.; Brüschweiler, R. J. Am. Chem. Soc. 1997, 119, 66726673.

(43) Lienin, S. F.; Bremi, T.; Brutscher, B.; Brüschweiler, R.; Ernst, R. R. J. Am. Chem. Soc. 1998, 120, 9870-9879.

(44) Bernado, P.; Blackledge, M. J. Am. Chem. Soc. 2004, 126, 77607761.

(45) Bouvignies, G.; Bernado, P.; Meier, S; Cho, K.; Grzesiek, S.; Brüschweiler, R.; Blackledge, M. Proc. Natl. Acad. Sci. U.S.A. 2005, 102, 13885-13890.

(46) Fadel, A. R.; Jin, D. Q.; Montelione, G. T.; Levy, R. M. J. Biomol. NMR 1995, 6, 221-226.

(47) Clore, G. M.; Schwieters, C. D. Biochemistry 2004, 43, 1067810691.

(48) Buck., M.; Karplus, M. J. Am. Chem. Soc. 1999, 121, 96459658.

(49) Friedland, G. D.; Lakomek, N. A.; Griesinger, C.; Meiler, J.; Kortemme, T. PLoS Comput. Biol 2009, 5, e1000393. 
(50) Shapiro, Yu. E.; Polimeno, A.; Freed, J. H.; Meirovitch, E. J. Phys. Chem. B 2011, 115, 354-365.

(51) Meirovitch, E.; Polimeno, A.; Freed, J. H. J. Phys. Chem. B 2006, 110, 20615-20628.

(52) Tugarinov, V.; Kay, L. E. J. Biomol. NMR 2004, 29, 369-376. 